\title{
Intracrine Vascular Endothelial Growth Factor Maintains Hippocampal Neural Stem Cell Quiescence
}

Tyler J. Dause ${ }^{1}$, Jiyeon K. Denninger ${ }^{1}$, Joshua D. Rieskamp², Akela A. Kuwahara ${ }^{3}$, Elizabeth D. Kirby ${ }^{1,4^{*}}$

1 Department of Psychology, College of Arts and Sciences, The Ohio State University, United States.

2 Neuroscience Graduate Program, The Ohio State University, United States

3 Department of Cell and Tissue Biology, University of California San Francisco, San Francisco, CA.

4 Chronic Brain Injury Initiative, The Ohio State University, United States

*Corresponding Author: kirby.224@osu.edu

\section{Summary}

In the dentate gyrus (DG) of the adult mouse hippocampus, neural stem cells (NSCs) balance self-renewal and differentiation to produce neurons that support hippocampal function. Vascular endothelial growth factor (VEGF) is a well-known supporting factor for adult neurogenesis, but conflicting studies have left it uncertain how VEGF signals to NSCs. Here, we identified a VEGF-VEGFR2 intracrine signaling mechanism within adult DG NSCs that prevents their exhaustion. We show both in vitro and in vivo that NSC-VEGF loss caused cell-autonomous exhaustion of adult DG NSCs. In contrast, extracellular VEGF was neither necessary nor sufficient to maintain NSC quiescence or to stimulate VEGFR2 signaling, most likely due to sheddase-mediated cleavage of extracellular VEGFR2 ligand binding domains. Our findings support an exclusively intracellular mechanism for VEGF signaling in adult DG NSCs, thereby providing resolution to previously conflicting studies and suggesting cellular source can dictate the functional impact of soluble ligands in DG NSCs.

\section{Introduction}

The dentate gyrus (DG) of the hippocampus is one of a few isolated regions in the adult mammalian brain where neural stem and progenitor cells (NSPCs) reside and proliferate throughout life (Bond et al., 2015). Balancing preservation of a quiescent radial glia-like neural stem cell (RGLNSC) pool and active proliferation of intermediate progenitors (IPCs) is essential for lifelong production of new neurons that support hippocampal function (Gage, 2019).

Vascular endothelial growth factor (VEGF) signaling has long been implicated in preservation of adult hippocampal neurogenesis. Early work by Fabel et al., 2003 and Cao et al., 2004 suggested that VEGF served as a primary molecular driver of cell proliferation in response to stimuli such as exercise and environmental enrichment. Subsequent studies from the late Ron Duman's lab more firmly implicated VEGF receptor 2 (VEGFR2) signaling in promoting NSPC proliferation both in healthy rodents (Fournier et al., 2012) and in rodent models of seizure (Segi-Nishida et al., 2008) and depression (Warner-Schmidt and Duman, 2007). We also previously showed that knockdown of VEGF specifically in adult NSPCs led to exhaustion of the DG RGL-NSC pool (Kirby et al., 2015), suggesting NSPC dependence specifically on selfsynthesized VEGF. However, more recently, several conflicting studies have emerged. Neutralization of VEGF in the DG was shown to have no effect on basal cell proliferation or neurogenesis (Licht et al., 2011) and the promotion of adult neurogenesis by
VEGF overexpression was shown to rely on indirect mechanisms such as angiogenesis or microglial activation (Kreisel et al., 2019; Licht et al., 2016).

Implicit in previous work, including our own, is the assumption that VEGF initiates signaling via activation of cell surface receptors after its secretion into the extracellular milieu. While VEGF is indeed a secreted, soluble protein, it also has been reported to maintain stemness in several non-neural stem cell populations in a cell autonomous fashion, signaling in an intracellular autocrine (intracrine) loop in cells that express both VEGF and VEGFR2 (Dias et al., 2000; Domigan et al., 2015; Gerber et al., 2002). How VEGF signals within the DG RGL-NSC population to regulate quiescence remains unclear and resolution of the multiple conflicting studies on the connection between VEGF and adult neurogenesis is lacking.

In this study, we investigate the cellular signaling mechanism by which VEGF maintains RGL-NSC quiescence both in cultured adult DG NSCs and within the adult rodent DG. We found that VEGF signaling maintains adult DG RGL-NSC quiescence exclusively through cell-internal VEGFR2 activation. Furthermore, we found that, unlike other stem cell populations that respond to both cell internal and cell external VEGF, DG NSCs actively cleaved VEGFR2 from their cell surface and were insensitive to extracellular VEGF levels. These findings help to reconcile previous contradictory findings based on their methodology targeting either intracellular VEGFR2 signaling within RGL-NSCs or extracellular VEGF availability. 


\section{Results}

NSC-VEGF initiates VEGFR2 signaling cellautonomously

We previously showed that loss of VEGF expression by adult DG RGL-NSCs and IPCs leads to RGL-NSC exhaustion at the population level despite only moderate $(\sim 30 \%)$ loss of total niche VEGF protein levels (Kirby et al., 2015). In contrast, several other studies showed no effect of more global and complete neutralization of extracellular VEGF on DG cell proliferation (Licht et al., 2011, 2016). These conflicting results raise the question of how a partial VEGF knockdown, targeting only RGLNSCs and IPCs, can impair NSC maintenance, while a more complete neutralization of extracellular VEGF has no effect. One possible explanation is that VEGF synthesized by RGL-NSCs signals cell autonomously through an intracellular autocrine (intracrine) loop in which VEGF binds to VEGFR2, the primary VEGF receptor, intracellularly.

To better understand the relative production of VEGF by DG RGL-NSCs, we analyzed several published single-cell RNA sequencing datasets derived from the adult mouse DG. We found that RGL-NSCs and their IPC progeny expressed Vegfa at levels intermediate between two other known producers of VEGF, astrocytes and endothelia (Figure 1A) (Batiuk et al., 2020; Hochgerner et al., 2018; Walker et al., 2020). RNAscope ${ }^{\circledR}$ in situ hybridization in adult mouse DG fixed tissue sections similarly confirmed that RGL-NSCs express Vegfa but $62.43 \pm 4.62 \%$ less than astrocytes (Figure 1B,C and S1A). Analysis of additional published RNAseq datasets revealed that hippocampal NSC Vegfa expression increases 1285 $\pm 45 \%$ from embryonic to adult ages (Berg et al., 2019) (S1B) and is $1254 \pm 102 \%$ greater in hippocampal NSPCs than in NSPCs from the SVZ (Adusumilli et al., 2021) (S1C). The same datasets also confirmed that hippocampal NSCs express $K d r$ (the transcript for VEGFR2) despite a decline in expression with age (S1D), and that $K d r$ expression is $524 \pm 23 \%$ higher in DG than in SVZ NSPCs (S1E). Finally, using RNAscope in situ hybridization, we directly confirmed that almost all (92.5\%) RGLNSCs co-expressed both Vegfa and Kdr within the same cell (Figure 1D, E, S1F). These findings imply that most RGL-NSCs have the potential for functional VEGF intracrine signaling and that high VEGF expression may be unique to adult DG RGLNSCs compared to developmental NSCs and adult SVZ NSCs.

To determine whether VEGFR2 intracrine signaling is active in NSCs, we examined phosphorylation of VEGFR2 downstream signaling pathways PI3K/Akt and MAPK/Erk in cultured NSCs derived from adult mouse DG. Intracellular VEGFR2 inhibition with either of two cell permeable inhibitors of VEGFR2 phosphorylation (SU5416 or SU1498) suppressed pAkt levels (Figure 1 F-G S1G-I) while pErk showed a more mild and transient fluctuation (Figure $1 \mathrm{H}$, S1G,I). In contrast, treatment with a VEGF antibody $(\mathrm{nAb})$ that specifically neutralizes extracellular VEGF did not alter pAkt or pErk (Figure 1I-K, S1JL). Both SU5416 and VEGF nAb blocked recombinant mouse VEGF-induced increases in pAkt and pErk in human umbilical vein endothelial cells (HUVECs), confirming that these reagents are bioactive and capable of preventing VEGF signaling through PI3K/Akt and MAPK/Erk pathways (S1M-R). Together, these data suggest that VEGFR2 signals through the PI3K/Akt pathway in NSCs and that intracrine VEGF signaling is sufficient to maintain this signaling in the absence of extracellular VEGF.

Cell autonomous NSC-VEGF is sufficient to maintain RGL-NSC quiescence in the adult DG

To determine if the cell autonomous VEGF signaling observed in vitro is sufficient to maintain quiescence in individual RGL-NSCs in vivo, we infused lentiviral vectors expressing GFP and a shRNA against Vegfa or a scramble shRNA control in the adult mouse DG. We then perfused mice 21or 60- days later, 2 hours after a single EdU injection to label mitotic cells (Figure 2A). In cultured NSCs, Vegfa shRNA led to a $60 \pm 11 \%$ suppression of secreted VEGF protein and $82 \pm 13 \%$ reduction in sphere number compared to scramble shRNA treated cells (S2A-C). In vivo, the lentiviral vectors showed wide tropism, as expected (S2D), and Vegfa shRNA reduced VEGF immunoreactivity throughout the DG by $74 \pm 12 \%$ compared to the scramble control (Figure 2B,C). Consistent with a stem cell exhaustion phenotype, Vegfa shRNA expression led to a significant increase in EdU+ GFP+ cell density at 21-days, followed by a near complete exhaustion of EdU+ GFP+ cells at 60 days, when compared to scramble control (Figure 2D,E). However, there were no significant changes in the neighboring GFPpopulation of EdU+ cells, indicating that the broad VEGF knockdown only impacted the GFP+ cell population. Similarly, by 60d, GFP+ RGL-NSCs were $75 \pm 6.6 \%$ depleted compared to scramble control while there was no effect of Vegfa shRNA expression on density of neighboring GFP- RGLNSCs (Figure 2F,G). Vegfa shRNA also significantly increased GFP+ IPC density after 21-days, and almost completely depleted GFP+ IPC density after 60-days compared to scramble control with no effect on neighboring GFP- IPCs (Figure 2H). No differences were found between Vegfa and scramble shRNA in neuronal phenotype of GFP+ or 

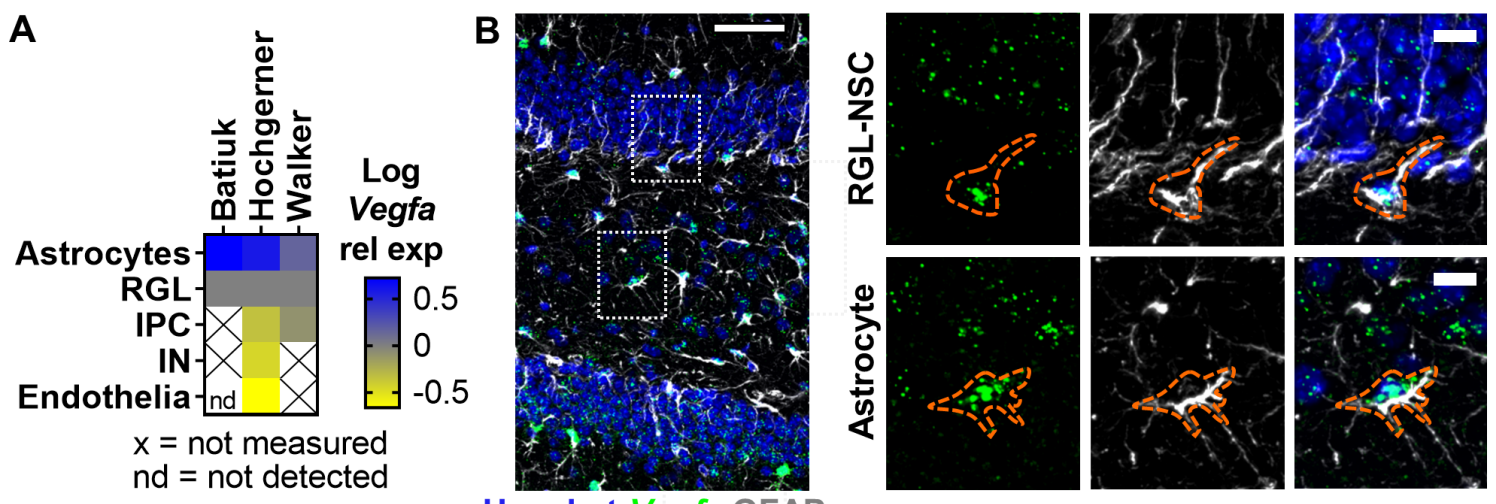

C
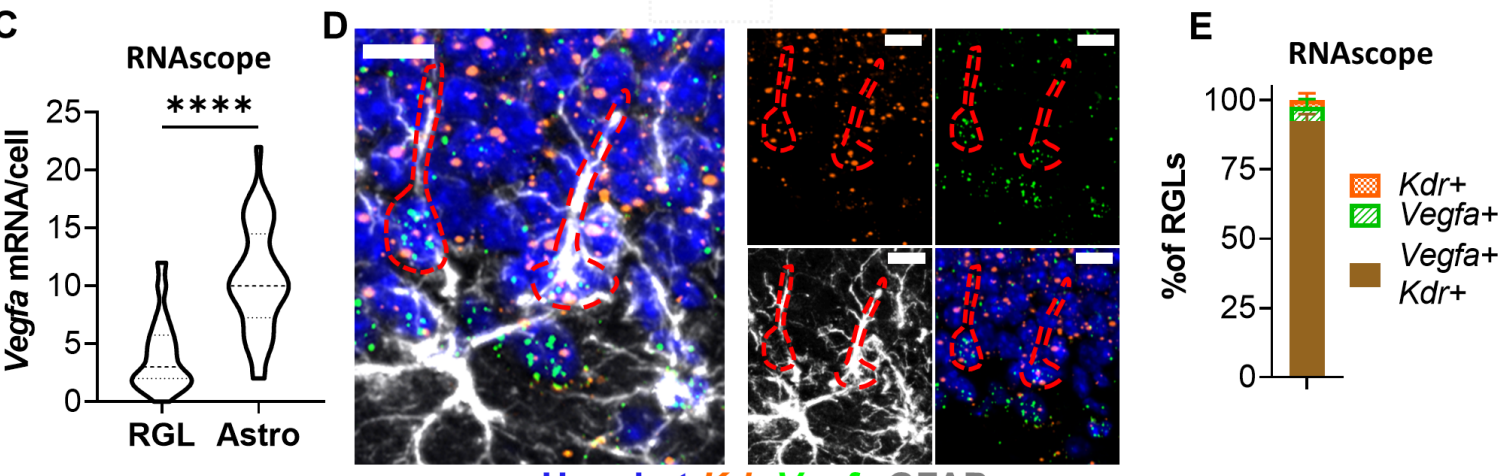

$\mathbf{F}$

SU5416

$\min 010153045$

pAkt - - -

Akt $-\cdots-\cdots$

pErk ニニニニニ

Erk こニニニニ

G
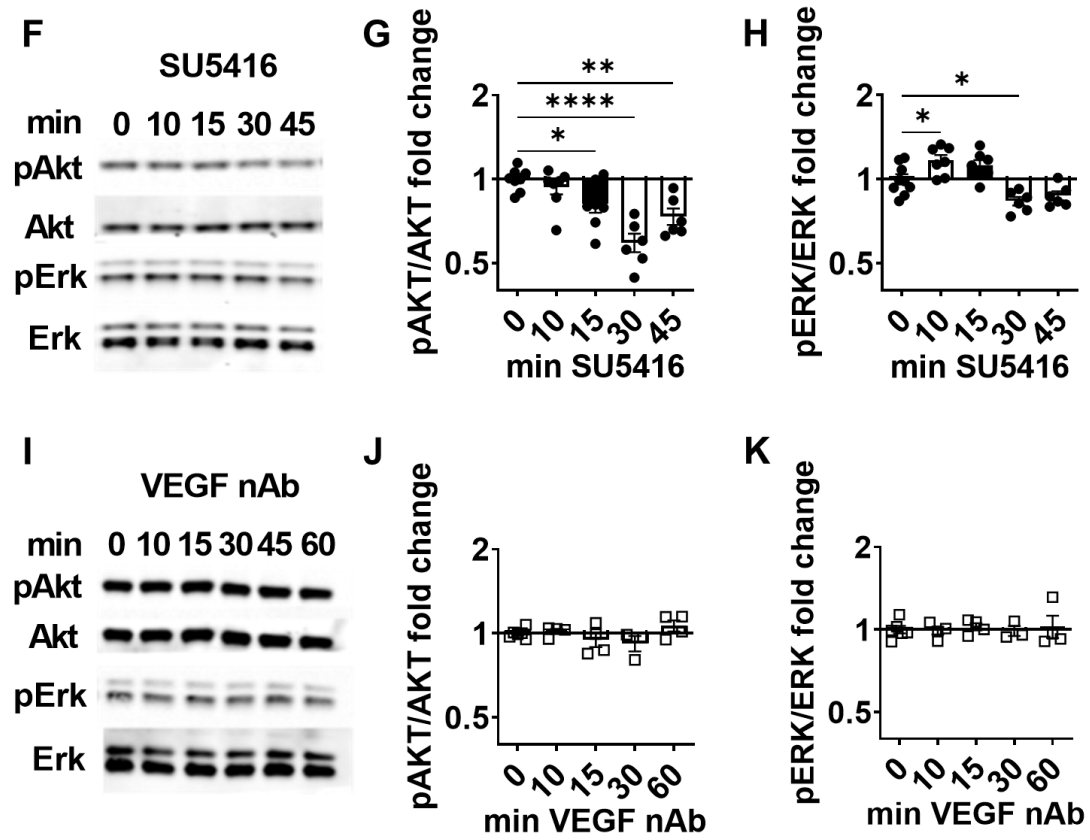

Figure 1: DG NSC-expressed VEGF signals via VEGFR2 cell-autonomously.

A) Heatmap of Vegfa expression from independently published single-cell RNA sequencing datasets (Batiuk et al., 2020; Hochgerner et al., 2018; Walker et al., 2020). IN = immature neurons

B) Vegfa in situ hybridization co-labeled with GFAP in adult mouse DG.

C) Vegfa RNA per cell. $\mathrm{N}=40$ cells/group; mean \pm SEM. Kolmogorov-Smirnov test.

D) $K d r$ and Vegfa in situ hybridization co-labeled with GFAP in adult mouse DG.

E) Percent of RGL-NSCs that are $K d r+$, Vegfa + or $K d r+/ V e g f a+. ~ N=4$ mice/grp; mean \pm SEM.

F, I) Representative western blots of Erk and Akt phosphorylation in cultured NSCs after SU5416 or VEGF nAb treatment.

$\mathrm{G}, \mathrm{J})$ Fold cP-hange in Akt or $\mathrm{H}, \mathrm{K}$ ) Erk phosphorylation after SU5416 or VEGF nAb, respectively. $\mathrm{N}=1$-3/grp/exp, 2 exps; mean \pm SEM. One-way ANOVA with Dunnett's multiple comparison test.

Scale bars represent B) $50 \mu \mathrm{M}$, subset $10 \mu \mathrm{M}$, D) $10 \mu \mathrm{M}$. ${ }^{* \star *} p<0.0001 ;{ }^{* *} p<0.01,{ }^{*} p<0.05$. nd, not detected. See also Figure S1. 

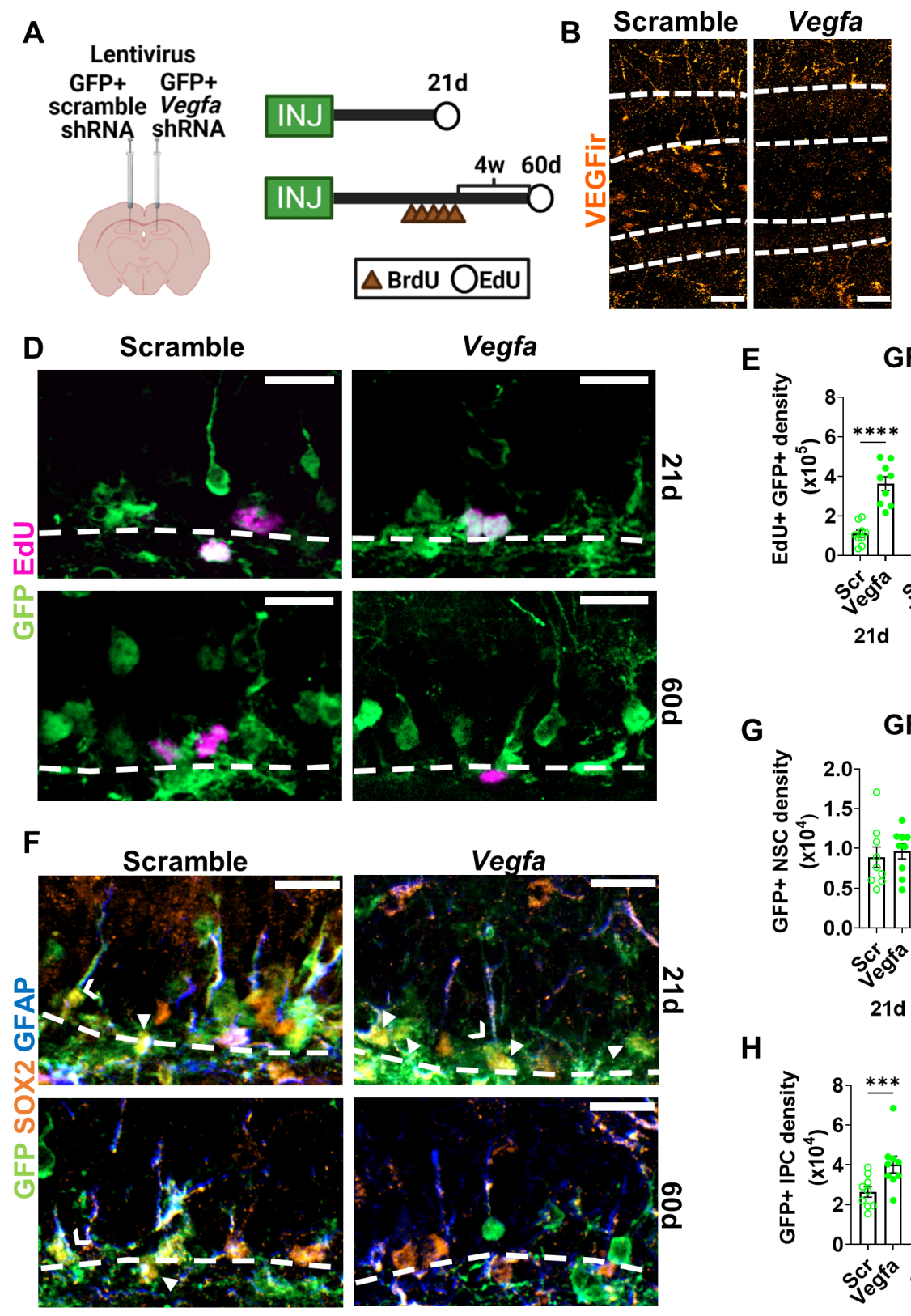

C

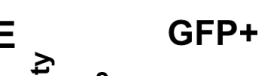

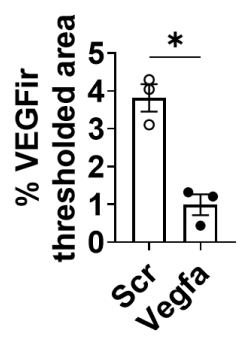

EdU+

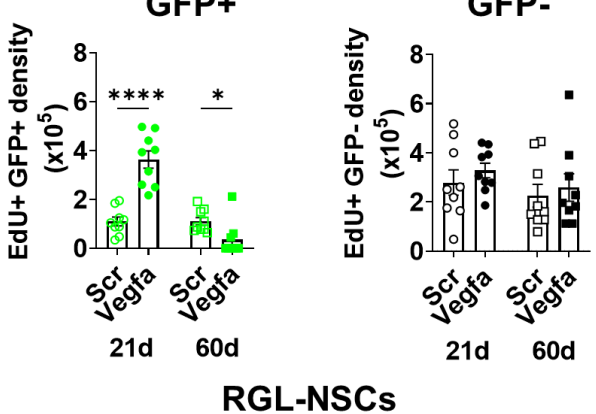

G

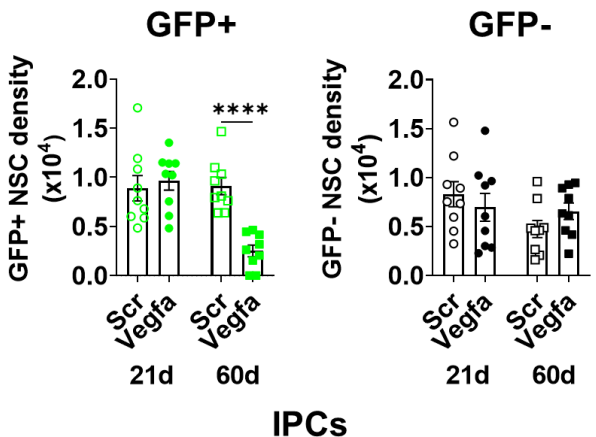

H
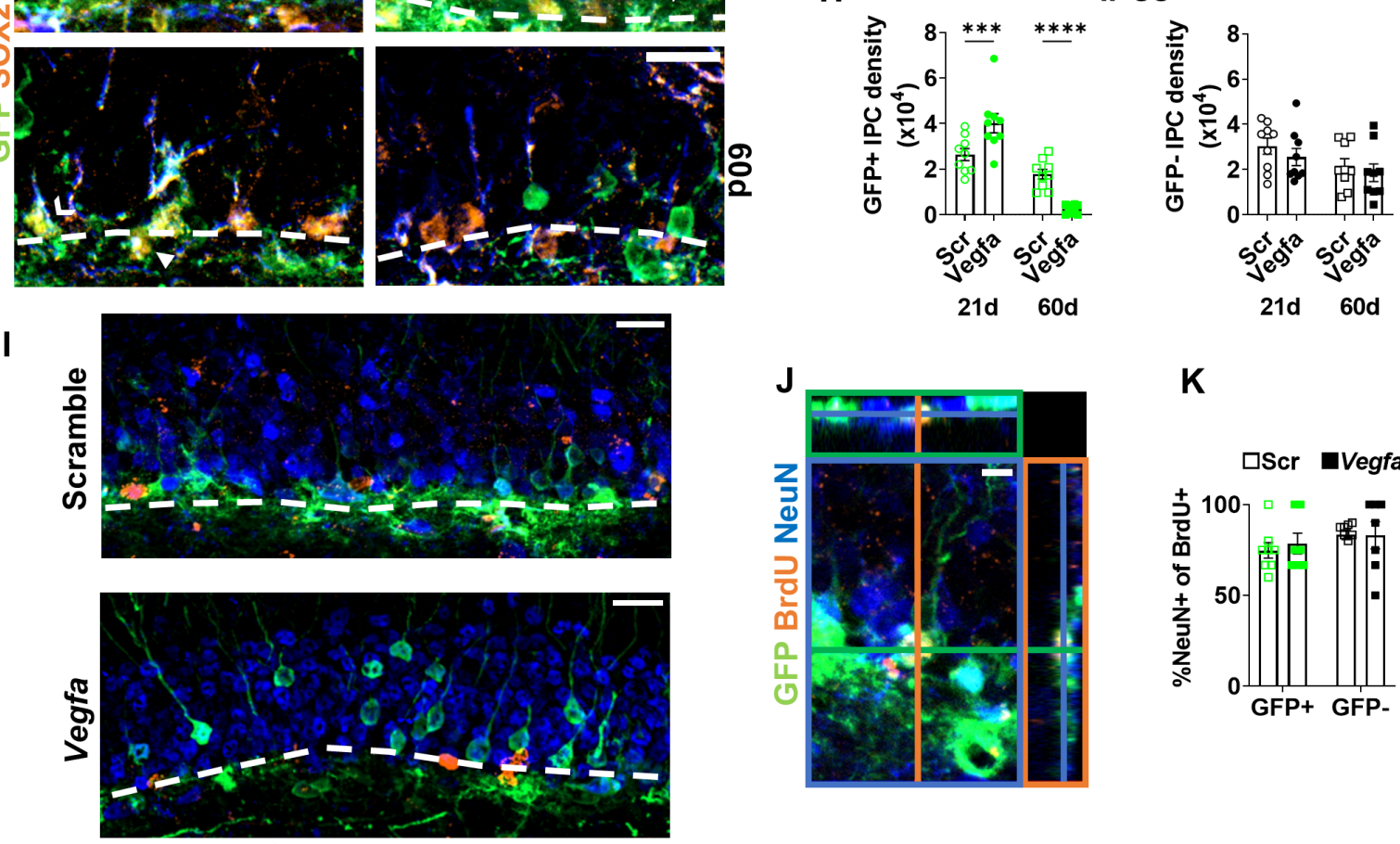

$\mathbf{K}$

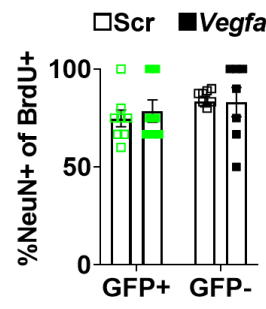

Figure 2: Cell autonomous NSC-VEGF is sufficient to maintain RGL-NSC quiescence in the adult DG

A) Diagram of experimental design and timeline.

B) Representative VEGF immunoreactivity in the DG 21d after viral infusion. Dashed line indicates granule cell layer.

C) Percent VEGF immunoreactive thresholded area in the DG $21 \mathrm{~d}$ after viral infusion. $\mathrm{N}=3$ mice/grp; mean \pm SEM. Ttest. 
D) Representative immunofluorescent images of shRNA expressing (GFP+) or non-expressing (GFP-) mitotically active $(E d U+)$ 21- and $60 d$ after viral infusion. Dashed line indicates subgranular zone-granule cell layer border.

E) Density of GFP+ or GFP- EdU+ cells $\left(\right.$ per $\mu \mathrm{m}^{2}$ ) after infection with scramble or Vegfa shRNA. N = 9 mice/grp; mean \pm SEM. Two-way ANOVA with Sidak's multiple comparison test.

F) Representative immunofluorescent images of shRNA expressing (GFP+) or non-expressing (GFP-) GFAP+ Sox2+ RGL-NSCs and GFAP-Sox2+ IPCs 21 and 60d after viral infusion. Chevrons indicate GFP+ RGL-NSCs, arrowheads indicate GFP+ IPCs.

G) Density of GFP+ or GFP- GFAP+ Sox2+ RGL-NSCs and H) GFAP-Sox2+ IPCs after infection with scramble or Vegfa shRNA 21 or $60 \mathrm{~d}$ after viral infusion. $\mathrm{N}=9 \mathrm{mice} / \mathrm{grp}$; mean \pm SEM. Two-way ANOVA with Sidak's multiple comparison test.

I) Representative immunofluorescent images of BrdU+ co-labeling with NeuN and GFP.

J) Orthogonal projection of a GFP+ BrdU+ NeuN+ cell.

K) Percent of BrdU+ cells that are NeuN+ among GFP+ and GFP- cells. N = 7-9 mice/grp; mean \pm SEM. Two-way ANOVA with Sidak's multiple comparison test.

Scale bars represent B) $10 \mu \mathrm{M}$ D, F, I) $20 \mu \mathrm{M}$ J) $5 \mu \mathrm{M} .{ }^{*} \mathrm{p}<0.05 ;{ }^{* *} \mathrm{p}<0.01 ;{ }^{* *} \mathrm{p}<0.001 ;{ }^{* * *} \mathrm{p}<0.0001$; See also Figure S2.

GFP- cells that were BrdU labeled 4 weeks before the $60 \mathrm{~d}$ harvest (Figure $2 \mathrm{I}-\mathrm{K}$ ), suggesting no strong impact of VEGF loss of new cell differentiation. Together, these data suggest that VEGF knockdown induces cell autonomous activation of RGL-NSCs, leading to a surge in IPC proliferation after 21 days and followed by exhaustion of the RGL-NSC pool at 60 days. The lack of effect of Vegfa shRNA expression on neighboring GFP- cells also implies that cell autonomous VEGF signaling is sufficient to maintain quiescence in these GFP- RGL-NSCs, even when extracellular VEGF is broadly eliminated.

\section{DG NSCs are insensitive to extracellular VEGF}

While the above findings suggest that cell autonomous VEGF signaling can maintain RGLNSCs, they do not clarify the role of extracellular VEGF signals derived from prominent paracrine sources such as astrocytes or even autocrine sources (i.e. other RGL-NSCs). To determine how paracrine (extracellular) VEGF regulates NSCs, we treated cultured NSCs with recombinant mouse VEGF. Neither pAkt nor pErk signaling were changed by VEGF treatment at any time point (Figure 3A-C) and increasing the extracellular VEGF treatment to $100 \mathrm{ng} / \mathrm{ml}$ VEGF (a supraphysiological dose) had no effect on pAkt or pErk (S3A-C). Depleting DG NSC media of standard supplemented EGF and FGF2 led to a rapid, partial suppression of pAkt and a complete elimination of detectable pErk (S3D-F), while feeding NSCs fresh EGF/FGF2 led to an increase in both pAkt and pErk (S3G-I). However, even in EGF/FGF2-depleted conditions, extracellular recombinant VEGF still did not alter pAkt signaling and pErk remained undetectable (S3J, K). These findings suggest that cultured NSCs do not respond to extracellular VEGF, even at supraphysiological doses or when competing signaling molecules are eliminated.

To determine whether extracellular VEGF could functionally signal in NSCs that had lost intracrine VEGF signaling, we used a neighbor rescue design (Figure 3D). We infected VEGFlox/lox or wildtype (WT) NSCs in vitro with a mCherry-Cre or mCherry-only lentiviral vector at a low multiplicity of infection (MOI). High MOI mCherry-Cre infection led to a significant suppression of VEGF in NSC conditioned media (S3L). At the low MOI, however, extracellular VEGF was not altered (S3M). Low MOI mCherry-Cre treatment led to an acute increase in BrdU+ proliferative cells selectively among mCherry+ cells while mCherry-only expression had no effect on proliferation (Figure 3E,F). When VEGFlox/lox NSCs were grown over multiple passages, a complete and selective loss of mCherry-Cre+ NSCs was observed while mCherryonly infected NSCs were preserved (Figure 3G,H). There was no effect of mCherry-Cre versus mCherry-only expression in WT NSCs (S3N-Q). These findings show that NSC activation and subsequent exhaustion after VEGF loss is cellautonomous and NSC reliance on cell-autonomous VEGF persists even when extracellular VEGF is high.

\section{Cell surface VEGFR2 is cleaved by sheddases}

The insensitivity of DG NSCs to extracellular VEGF suggests that VEGFR2 may not be at the cell surface as expected. Using immunocytochemical staining of non-permeabilized and permeabilized cultured DG NSCs, we confirmed that only permeabilized NSCs showed immunoreactivity with an antibody targeting the N-terminus of VEGFR2, suggesting that the N-terminal VEGF binding sites of VEGFR2 are present only within the intracellular compartment of cultured NSCs (Figure 4A). In contrast, mouse brain endothelial cells (bEnd.3), which are well established to respond to extracellular VEGF, showed N-terminal VEGFR2immunoreactivity both on the cell surface and within the intracellular compartment (S4A). CRISPRi suppression of $\mathrm{Kdr}$ expression ( $\alpha \mathrm{VEGFR2,} \mathrm{S4B)}$ significantly reduced both intracellular and extracellular VEGFR2 immunoreactivity in b.End3 cells and intracellular VEGFR2 immunoreactivity in 


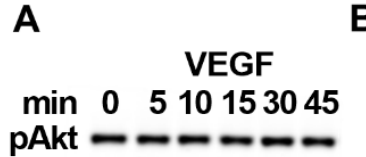

Akt $-\cdots-\cdots-\cdots$

pErk $=-=-=-$
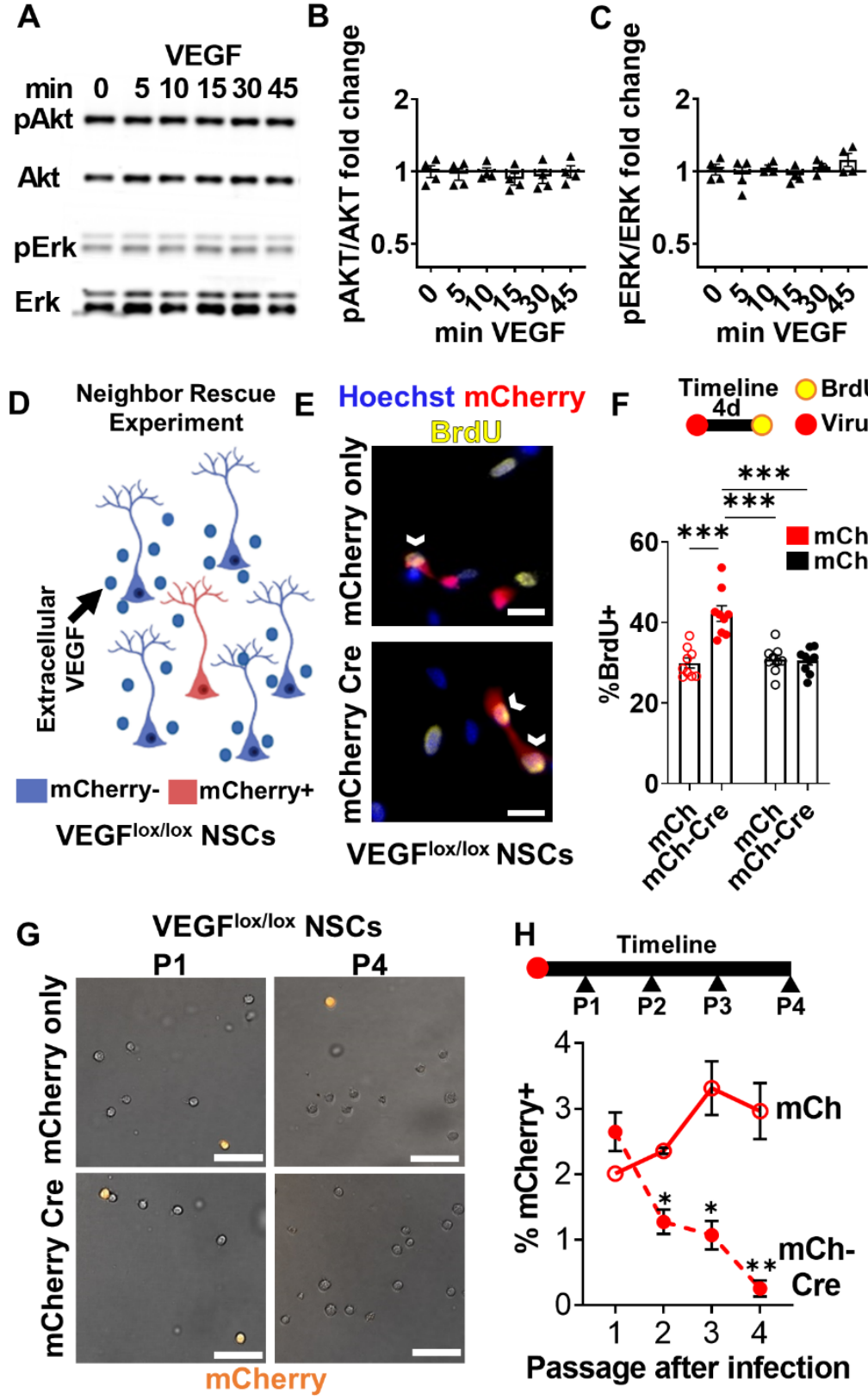

Figure 3: NSC VEGFR2 signaling and quiescence are insensitive to extracellular VEGF

A) Representative western blot of Erk and Akt phosphorylation in cultured DG NSCs after treatment with recombinant VEGF.

B) Fold change in Akt or C) Erk phosphorylation after $10 \mathrm{ng} / \mathrm{ml}$ VEGF treatment in cultured DG NSCs. $\mathrm{N}=2 / \mathrm{grp} / \mathrm{exp}, 2$ exps; mean \pm SEM. One-way ANOVA with Dunnett's multiple comparison test.

D) Diagram of the Neighbor Rescue Experiment. VEGFloxlox NSCs infected with mCherry-Cre virus will undergo VEGF knockdown, but be exposed to extracellular VEGF from neighboring mCherry- NSCs.

E) Representative images of mitotically active (BrdU+) cultured DG VEGFlox/lox NSCs (Hoechst+) after lentiviral infection (mCherry+). Chevrons indicate BrdU+, mCherry+ Hoechst + NSCs.

F) Percent of mCherry+ or mCherry- VEGFloxlox NSCs that were BrdU+ after low $\mathrm{MOI}$ mCherry-Cre and mCherry-only lentiviral infection. $\mathrm{N}=3 / \mathrm{grp} / \mathrm{exp}, 3$ exps; mean \pm SEM. Two-way ANOVA with Tukey's multiple comparisons test.

G) Representative images of cultured VEGFlox/lox NSCs (brightfield) after lentiviral infection (mCherry+) after 1 and 4 passages.

H) Percent of mCherry+ VEGFlox/lox NSCs after infection with low MOI mCherryCre or mCherry-only lentiviral vectors. $\mathrm{N}=3 /$ grp/exp, 2 exps; mean $\pm \mathrm{SEM}$. Twoway ANOVA with Sidak's multiple comparisons test.

Scale bars represent E) $10 \mu \mathrm{M}$ G) $50 \mu \mathrm{M}$. ${ }^{*} p<0.05 ;{ }^{* *} p<0.01 ;{ }^{* * *} p<0.001$. See also Figure S3.
NSCs compared to a non-target single guide RNA (NT) (S4C-E), confirming the specificity of the VEGFR2 antibody.

Because VEGFR2 was not detectable on the cell surface of cultured NSCs, we chose to investigate whether VEGFR2 is being rapidly cleaved by sheddases after insertion into the membrane. Sheddases are membrane-bound enzymes that cleave trans-membrane proteins, consisting of members from the ADAM, BACE and MMP protein families. Analysis of our own published single-cell RNA sequencing and liquid chromatography tandem mass spectrometry data from cultured adult DG NSCs revealed that multiple sheddases are detectable throughout the cell cycle and in quiescent NSCs, particularly Mmp15, Adam9, Adam10 and Adam12 (Figure 4B,C) (Denninger et al., 2020). Multiple sheddases were also detected in single cell RNAseq of in vivo DG RGL-NSCs from (Shin et al., 2015) (Figure 4D). Treating cultured NSCs with tumor necrosis factor- $\alpha$ protease inhibitor 1 (TAPI-1), a broadspectrum inhibitor of sheddases, significantly increased VEGFR2 immunoreactivity on the cell surface of cultured NSCs (Figure 4E,F), suggesting that NSC-expressed sheddases actively cleave VEGFR2 from the cell surface, thereby preventing NSC response to extracellular VEGF.

\section{Discussion}

Regulation of the balance between NSC activation and quiescence is critical to preserve the production of adult born neurons in the adult hippocampus. Here, we identified a novel mechanism by which adult DG NSCs use selfexpressed, cell autonomous VEGF to maintain their own quiescence using cell internal activation of VEGFR2 while actively cleaving VEGFR2 from the cell surface and remaining unresponsive to extracellular VEGF levels. Our findings therefore suggest that cellular source and intracellular receptor location are critical factors to consider when assessing the effect of soluble proteins on NSCs.

The VEGF autocrine signaling mechanism that we describe here appears to be somewhat unique to NSCs of the adult DG. Though embryonic NSCs are well-known to produce and secrete VEGF ligand (Komabayashi-Suzuki et al., 2019), they do not rely on intracrine or autocrine VEGFR signaling for self-regulation (Han et al., 2015; Wada et al., 2006). Rather, embryonic NSC VEGF seems to be critical primarily for stimulating proliferation 

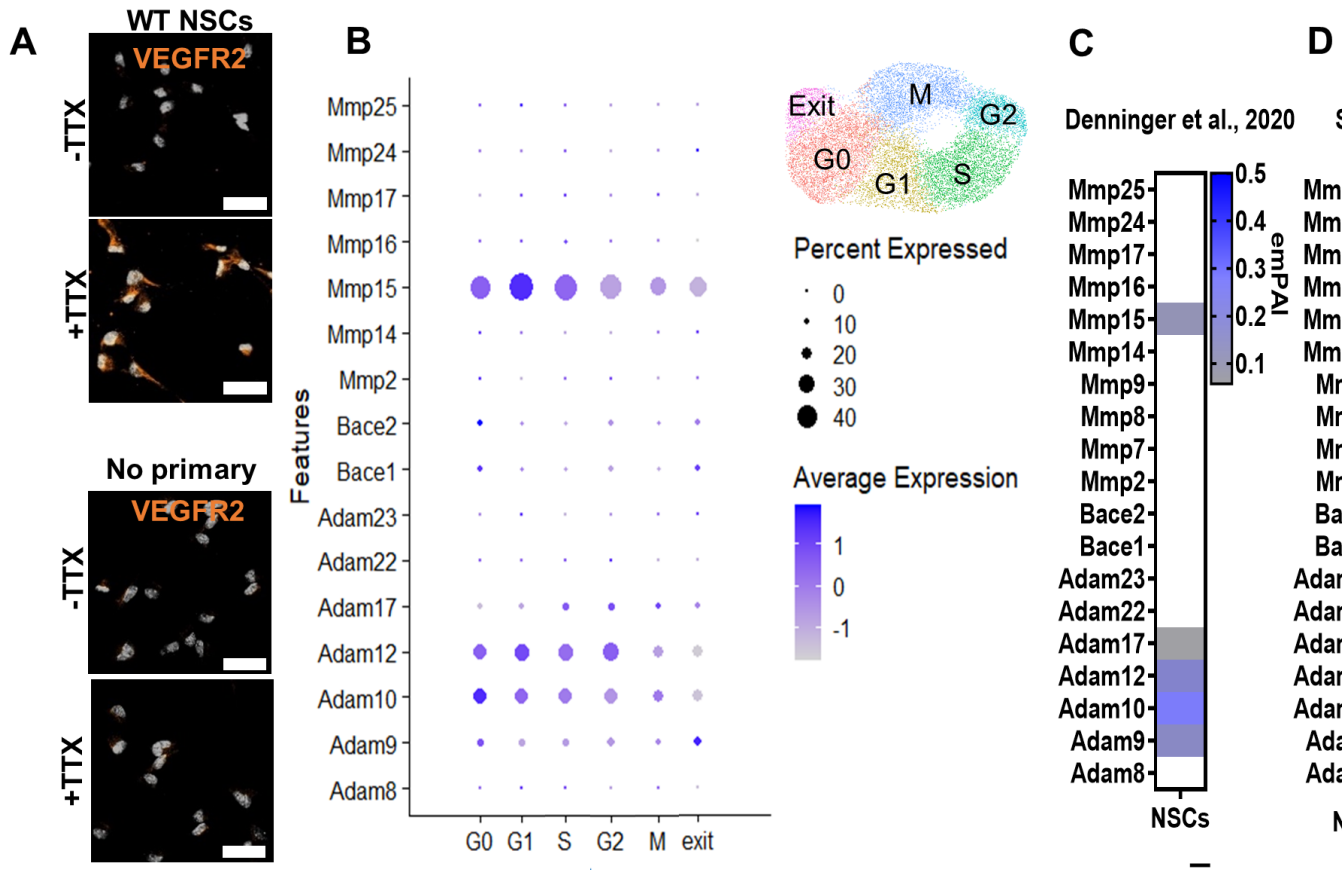

Denninger et al., 2020 Shin et al., 2015
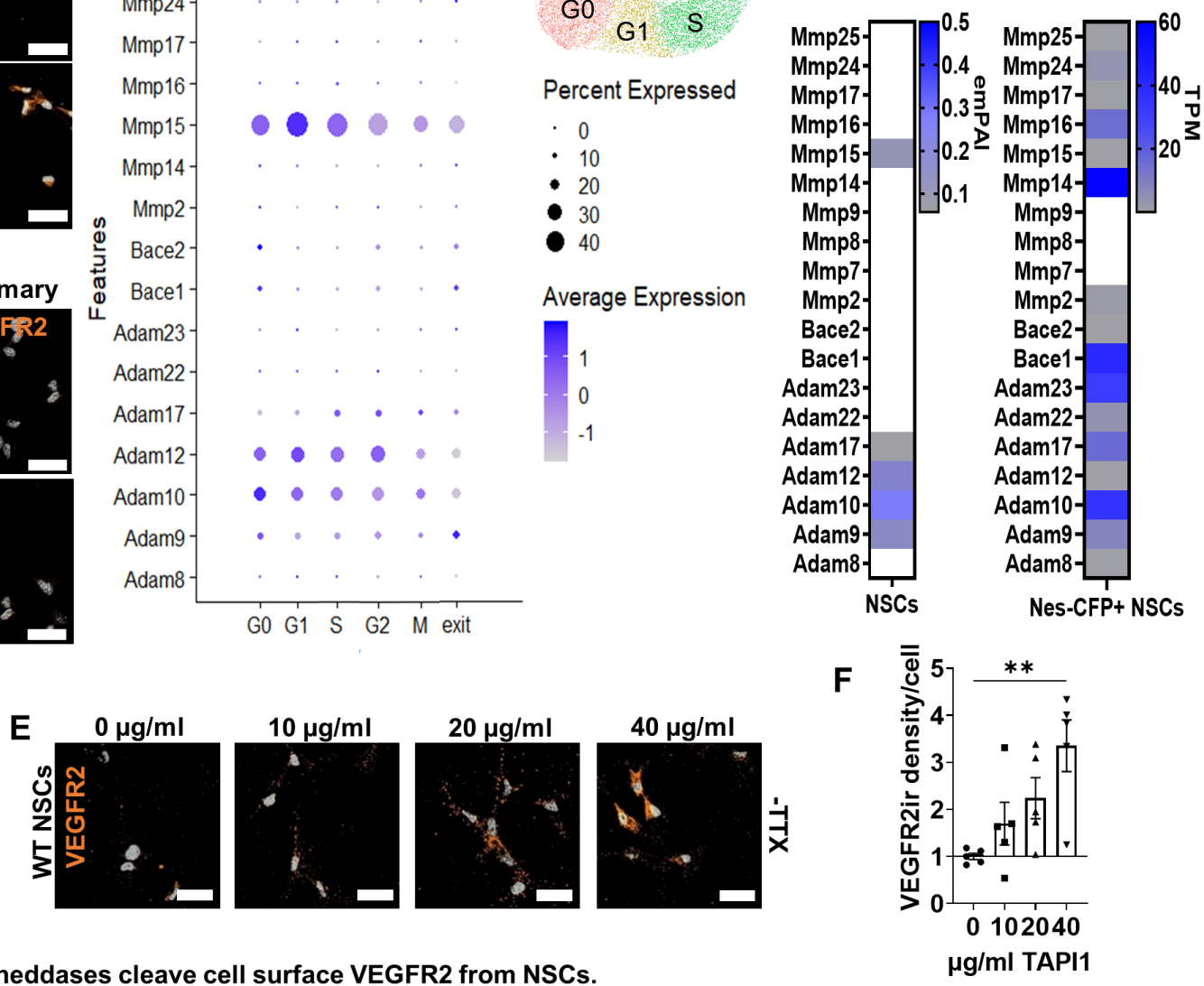

Figure 4: Sheddases cleave cell surface VEGFR2 from NSCs.

$\mu \mathrm{g} / \mathrm{ml}$ TAPI1

A) VEGFR2 immunoreactivity of permeabilized and non-permeabilized cultured DG NSCs in VEGFR2 primary and no primary conditions.

B) Sheddase RNA counts from single cell RNAseq and C) protein expression (emPAI) from LC-MS/MS of cultured DG NSCs in Denninger et al., 2020.

D) Sheddase RNA expression (TPM) from single cell RNA sequencing data derived from acutely isolated adult hippocampal NSCs from Shin et al., 2015.

E) Representative VEGFR2 immunoreactivity in non-permeabilized NSCs following TAPI-1 treatment in cultured NSCs.

F) Mean cell surface VEGFR2 immunoreactivity per cell following TAPI-1 treatment in cultured NSCs. N = 2 wells/exp,

2 exps; mean \pm SEM. One-way ANOVA with Kruskal-Wallis test.

Scale bars represent $A, E) 20 \mu \mathrm{M} .{ }^{* *} p<0.01$. See also Figure $S 4$.

and migration of developing vasculature (Komabayashi-Suzuki et al., 2019). It also seems unlikely that NSCs in the adult SVZ rely on VEGF intracrine signaling as we found here that adult SVZ NSCs express much lower levels of VEGF and VEGFR2 than DG NSCs. The mechanisms by which NSCs in the DG develop reliance on VEGF intracrine signaling to maintain quiescence remain to be investigated, as does the possibility that this shift supports their deepening quiescence over postnatal development (Urbán et al., 2019).

VEGF has previously been shown to support survival and/or quiescence through an intracrine loop in several other cell populations, including hematopoietic stem cells, cancer stem cells and endothelia (Dias et al., 2000; Domigan et al., 2015; Gerber et al., 2002). In addition to intracrine signaling loops, however, these cells are typically also sensitive to extracellular VEGF (Wiszniak and Schwarz, 2021). In contrast, we found that DG NSCs were insensitive to extracellular VEGF levels, likely because the extracellular VEGF binding domain of VEGFR2 is cleaved by sheddases on the cell surface. This insensitivity to extracellular VEGF provides substantial resolution for the previously conflicting literature on VEGF and adult neurogenesis. Specifically, studies suggesting no effect of VEGF loss on adult neurogenesis often relied on methods that neutralized extracellular VEGF (Licht et al., 2011, 2016) while studies suggesting that loss of VEGF signaling suppressed adult neurogenesis often relied on long-term infusion of cell permeable VEGFR2 inhibitors (Fournier et al., 2012; Segi-Nishida et al., 2008; Warner-Schmidt and Duman, 2007). All of these results are consistent with a model where cell internal VEGF is necessary and sufficient to prevent RGL-NSC exhaustion and external VEGF does not directly impact RGL-NSCs. 
Determining the key mechanisms by which NSCs self-regulate is an important step in understanding the preservation of adult hippocampal neurogenesis. Our findings suggest that VEGF regulation of adult NSC maintenance depends critically on the source of the VEGF ligand. These findings have important implications when considering how to therapeutically support endogenous adult neurogenesis or exogenous NSC transplants.

\section{Limitations of the Study}

There are several limitations to the present study. First, though we have previously shown VEGF protein production by NSPCs as a population (Kirby et al., 2015), our analysis of VEGF and VEGFR2 coexpression in single RGL-NSCs here relies on transcriptional-level analysis. Identification of the cellular source of VEGF protein is complicated by the fact that secreted proteins have short intracellular half-lives and most immunolabeling for VEGF is therefore found in the interstitial space or bound to/inside receptor expressing cells. VEGFR2 antibodies in vivo have also proven unreliable (Licht et al., 2016). Second, VEGF-A binds to several receptors and co-receptors in the rodent brain. While VEGFR2 is the primary receptor, it is possible that NSC-specific VEGF may also signal through other NSC-expressed VEGF receptors to maintain quiescence or may also rely on binding to coreceptors (e.g. neuropilins) (Koch and ClaessonWelsh, 2012). Further study of these receptors, coreceptors and downstream signaling pathways are necessary to confirm the molecular mechanism by which VEGF signals to maintain NSC quiescence. Third, in our experiments we use rodent models to study VEGF signaling in adult hippocampal NSCs. Additional experiments are needed to determine if human NSCs use cell autonomous VEGF signaling to maintain quiescence.

Funding: NSF IOS-1923094 to EDK. NIH/NCI Center Core Grant P30CA016058 to the OSU Comprehensive Cancer Center/Dr. Michael A. Caligiuri.

\section{References}

Adusumilli, V.S., Walker, T.L., Overall, R.W., Klatt, G.M., Zeidan, S.A., Zocher, S., Kirova, D.G., Ntitsias, K., Fischer, T.J., Sykes, A.M., et al. (2021). ROS Dynamics Delineate Functional States of Hippocampal Neural Stem Cells and Link to Their Activity-Dependent Exit from Quiescence. Cell Stem Cell 28, 300-314.e6.

Babu, H., Claasen, J.-H., Kannan, S., Rünker, A.E., Palmer, T., and Kempermann, G. (2011). A protocol for isolation and enriched monolayer cultivation of neural precursor cells from mouse dentate gyrus. Front. Neurosci. 5, 89.

Batiuk, M.Y., Martirosyan, A., Wahis, J., de Vin, F., Marneffe, C., Kusserow, C., Koeppen, J., Viana, J.F., Oliveira, J.F., Voet, T., et al. (2020). Identification of region-specific astrocyte subtypes at single cell resolution. Nat. Commun. 11, 1220.

Berg, D.A., Su, Y., Jimenez-Cyrus, D., Patel, A., Huang, N., Morizet, D., Lee, S., Shah, R., Ringeling, F.R., Jain, R., et al. (2019). A Common Embryonic Origin of Stem Cells Drives Developmental and Adult Neurogenesis. Cell 177, 654-668.e15.

Bond, A.M., Ming, G., and Song, H. (2015). Adult Mammalian Neural Stem Cells and Neurogenesis: Five Decades Later. Cell Stem Cell 17, 385-395.

Cao, L., Jiao, X., Zuzga, D.S., Liu, Y., Fong, D.M., Young, D., and During, M.J. (2004). VEGF links hippocampal activity with neurogenesis, learning and memory. Nat. Genet. 36, 827-835.

Chiou, S.-H., Winters, I.P., Wang, J., Naranjo, S., Dudgeon, C., Tamburini, F.B., Brady, J.J., Yang, D., Grüner, B.M., Chuang, C.-H., et al. (2015). Pancreatic cancer modeling using retrograde viral vector delivery and in vivo CRISPR/Cas9-mediated somatic genome editing. Genes Dev. 29, 1576-1585.

Denninger, J.K., Chen, X., Turkoglu, A.M., Sarchet, P., Volk, A.R., Rieskamp, J.D., Yan, P., and Kirby, E.D. (2020). Defining the adult hippocampal neural stem cell secretome: In vivo versus in vitro transcriptomic differences and their correlation to secreted protein levels. Brain Res. 1735, 146717. 
Dias, S., Hattori, K., Zhu, Z., Heissig, B., Choy, M., Lane, W., Wu, Y., Chadburn, A., Hyjek, E., Gill, M., et al. (2000). Autocrine stimulation of VEGFR-2 activates human leukemic cell growth and migration. J. Clin. Invest. $106,511-521$.

Domigan, C.K., Warren, C.M., Antanesian, V., Happel, K., Ziyad, S., Lee, S., Krall, A., Duan, L., TorresCollado, A.X., Castellani, L.W., et al. (2015). Autocrine VEGF maintains endothelial survival through regulation of metabolism and autophagy. J. Cell Sci. 128, 2236-2248.

Fabel, K., Fabel, K., Tam, B., Kaufer, D., Baiker, A., Simmons, N., Kuo, C.J., and Palmer, T.D. (2003). VEGF is necessary for exercise-induced adult hippocampal neurogenesis. Eur. J. Neurosci. 18, 2803-2812.

Fournier, N.M., Lee, B., Banasr, M., Elsayed, M., and Duman, R.S. (2012). Vascular endothelial growth factor regulates adult hippocampal cell proliferation through MEK/ERK- and PI3K/Akt-dependent signaling.

Neuropharmacology 63, 642-652.

Gage, F.H. (2019). Adult neurogenesis in mammals. Science 364, 827-828.

Gerber, H.-P., Malik, A.K., Solar, G.P., Sherman, D., Liang, X.H., Meng, G., Hong, K., Marsters, J.C., and Ferrara, N. (2002). VEGF regulates haematopoietic stem cell survival by an internal autocrine loop mechanism. Nature 417, 954-958.

Han, J., Calvo, C.-F., Kang, T.H., Baker, K.L., Park, J.-H., Parras, C., Levittas, M., Birba, U., Pibouin-Fragner, L., Fragner, P., et al. (2015). Vascular Endothelial Growth Factor Receptor 3 Controls Neural Stem Cell Activation in Mice and Humans. Cell Rep. 10, 1158-1172.

Hochgerner, H., Zeisel, A., Lönnerberg, P., and Linnarsson, S. (2018). Conserved properties of dentate gyrus neurogenesis across postnatal development revealed by single-cell RNA sequencing. Nat. Neurosci. 21, 290299.

Keyoung, H.M., Roy, N.S., Benraiss, A., Louissaint, A., Suzuki, A., Hashimoto, M., Rashbaum, W.K., Okano, H., and Goldman, S.A. (2001). High-yield selection and extraction of two promoter-defined phenotypes of neural stem cells from the fetal human brain. Nat. Biotechnol. 19, 843-850.

Kirby, E.D., Kuwahara, A.A., Messer, R.L., and Wyss-Coray, T. (2015). Adult hippocampal neural stem and progenitor cells regulate the neurogenic niche by secreting VEGF. Proc. Natl. Acad. Sci. U. S. A. 112, 41284133.

Koch, S., and Claesson-Welsh, L. (2012). Signal transduction by vascular endothelial growth factor receptors. Cold Spring Harb. Perspect. Med. 2, a006502.

Komabayashi-Suzuki, M., Yamanishi, E., Watanabe, C., Okamura, M., Tabata, H., Iwai, R., Ajioka, I., Matsushita, J., Kidoya, H., Takakura, N., et al. (2019). Spatiotemporally Dependent Vascularization Is Differently Utilized among Neural Progenitor Subtypes during Neocortical Development. Cell Rep. 29, 11131129.e5.

Kreisel, T., Wolf, B., Keshet, E., and Licht, T. (2019). Unique role for dentate gyrus microglia in neuroblast survival and in VEGF-induced activation. Glia 67, 594-618.

Licht, T., Goshen, I., Avital, A., Kreisel, T., Zubedat, S., Eavri, R., Segal, M., Yirmiya, R., and Keshet, E. (2011). Reversible modulations of neuronal plasticity by VEGF. Proc. Natl. Acad. Sci. U. S. A. 108, 5081-5086.

Licht, T., Rothe, G., Kreisel, T., Wolf, B., Benny, O., Rooney, A.G., Ffrench-Constant, C., Enikolopov, G., and Keshet, E. (2016). VEGF preconditioning leads to stem cell remodeling and attenuates age-related decay of adult hippocampal neurogenesis. Proc. Natl. Acad. Sci. U. S. A. 113, E7828-E7836. 
Mosher, K.I., Andres, R.H., Fukuhara, T., Bieri, G., Hasegawa-Moriyama, M., He, Y., Guzman, R., and WyssCoray, T. (2012). Neural progenitor cells regulate microglia functions and activity. Nat. Neurosci. 15, 14851487.

Pan, Q., Chanthery, Y., Liang, W.-C., Stawicki, S., Mak, J., Rathore, N., Tong, R.K., Kowalski, J., Yee, S.F., Pacheco, G., et al. (2007). Blocking neuropilin-1 function has an additive effect with anti-VEGF to inhibit tumor growth. Cancer Cell 11, 53-67.

Segi-Nishida, E., Warner-Schmidt, J.L., and Duman, R.S. (2008). Electroconvulsive seizure and VEGF increase the proliferation of neural stem-like cells in rat hippocampus. Proc. Natl. Acad. Sci. 105, 1135211357.

Shin, J., Berg, D.A., Zhu, Y., Shin, J.Y., Song, J., Bonaguidi, M.A., Enikolopov, G., Nauen, D.W., Christian, K.M., Ming, G., et al. (2015). Single-Cell RNA-Seq with Waterfall Reveals Molecular Cascades underlying Adult Neurogenesis. Cell Stem Cell 17, 360-372.

Thakore, P.I., D'Ippolito, A.M., Song, L., Safi, A., Shivakumar, N.K., Kabadi, A.M., Reddy, T.E., Crawford, G.E., and Gersbach, C.A. (2015). Highly specific epigenome editing by CRISPR-Cas9 repressors for silencing of distal regulatory elements. Nat. Methods 12, 1143-1149.

Urbán, N., Blomfield, I.M., and Guillemot, F. (2019). Quiescence of Adult Mammalian Neural Stem Cells: A Highly Regulated Rest. Neuron 104, 834-848.

Wada, T., Haigh, J.J., Ema, M., Hitoshi, S., Chaddah, R., Rossant, J., Nagy, A., and van der Kooy, D. (2006). Vascular endothelial growth factor directly inhibits primitive neural stem cell survival but promotes definitive neural stem cell survival. J. Neurosci. Off. J. Soc. Neurosci. 26, 6803-6812.

Walker, L.A., Sovic, M.G., Chiang, C.-L., Hu, E., Denninger, J.K., Chen, X., Kirby, E.D., Byrd, J.C., Muthusamy, N., Bundschuh, R., et al. (2020). CLEAR: coverage-based limiting-cell experiment analysis for RNA-seq. J. Transl. Med. 18, 63.

Warner-Schmidt, J.L., and Duman, R.S. (2007). VEGF is an essential mediator of the neurogenic and behavioral actions of antidepressants. Proc. Natl. Acad. Sci. 104, 4647-4652.

Wiszniak, S., and Schwarz, Q. (2021). Exploring the Intracrine Functions of VEGF-A. Biomolecules 11, 128. 


\section{Methods}

Resource Availability

\section{Lead Contact}

Further information and requests for resources and reagents should be directed to the lead contact Dr. Elizabeth

Kirby, kirby.224@osu.edu

\section{Materials availability}

NT and VEGFR2 CRISPRi plasmids generated in this study will be deposited to Addgene.

\section{Data and code availability}

This paper analyzes existing, publicly available data. These accession numbers for the datasets are listed in the key resources table. All data reported in this paper will be shared by the lead contact upon request. Any additional information required to reanalyze the data reported in this paper is available from the lead contact upon request.

\section{Experimental Model and Subject Details Mice}

All animal use was in accordance with institutional guidelines approved by The Ohio State University Institutional Animal Care and Use Committee. Wild type C57/BI6J male and female mice were purchased from Jackson Laboratory and housed in standard ventilated cages, with ad libitum access to food and water throughout all experiments and maintained on a $12 \mathrm{~h}$ light cycle with lights on at $630 \mathrm{~h}$. Male and female mice were represented in approximately equal numbers throughout and no differences in sex were found. We therefore combined them in all presented analysis.

\section{NSC isolation}

NSCs were isolated from adult hippocampus of C57BL/6J or VEGFloxlox mice (described in Kirby et al., 2015) as described in Babu et al., 2011. Unless otherwise stated, NSCs were maintained on poly-D-lysine (Sigma) and laminin (Invitrogen) coated plates in Neurobasal A media (Invitrogen) with 1x B27 supplement without vitamin A (GIBCO), 1x glutamax (Invitrogen) and $20 \mathrm{ng} / \mathrm{ml}$ each of EGF and FGF2 (Peprotech), as per (Babu et al., 2011). No cells were used past passage 20. Two separate lines were used in all experiments, one from 5 pooled C57/BI6J male mice and one from 5 pooled C57/BI6J female mice. No differences between NSCs isolated from males and females were found.

\section{Endothelial Cells}

Human Umbilical Vein Endothelial Cells (HUVEC, Invitrogen) were maintained as per manufacturer instructions on uncoated tissue culture plates.

bEnd.3 cells (ATCC CRL-2299) were maintained in Dulbecco's Modified Eagle's Medium (ATCC 30-2002) with $10 \%$ fetal bovine serum (Fisher $10-437-028$ ) at $37{ }^{\circ} \mathrm{C}, 5 \% \mathrm{CO}_{2}$ in tissue culture dishes.

\section{Methods Detail}

RNAscope in situ hybridization and immunohistochemistry. WT C57BL/6J mice were transcardially perfused with ice cold PBS followed by $4 \%$ PFA. Brains were harvested and fixed overnight at $4{ }^{\circ} \mathrm{C}$ in $4 \%$ PFA before serial overnight equilibration in 10\%, 20\%, and 30\% sucrose. Fixed tissue was snap frozen in OCT in a dry ice $/ 100 \%$ ethanol bath and stored at $-70^{\circ} \mathrm{C}$. $12 \mu \mathrm{m}$ cryosections, 1 section per slide, were prepared with a cryostat and stored at $-70^{\circ} \mathrm{C}$ with desiccant until staining. RNA in situ hybridization was performed according to manufacturer recommendations for using fixed frozen tissue samples in the RNAscope Multiplex Fluorescent v2 Assay (Advanced Cell Diagnostics) with the following modifications to enable concurrent immunohistochemical staining. The pretreatment steps were replaced with a 15 min modified citrate buffer (Dako) antigen retrieval step in a steamer at $95^{\circ} \mathrm{C}$. Additionally, the protease III step was excluded to enable subsequent immunohistochemical staining. Probes for mouse Vegfa (Mm-Vegfa-ver2, ACD 412261) and mouse Kdr (MmKdr-C2, ACD 414811-C2) RNA were hybridized to tissue before subsequent immunohistochemical staining for GFAP protein. Immunostaining for GFAP was conducted as described in (Immunohistochemical tissue processing) with the following exceptions. Blocking was performed with $10 \%$ normal donkey serum in TBS-1\% BSA. Antibody incubations were performed in TBS-1\% BSA. All washes were performed with TBST. DAPI provided by the RNAscope Multiplex Fluorescent kit was used for nuclear counterstaining. All images were acquired with the Zeiss Axio Observer Z1 microscope with Apotome for optical sectioning using a 20x air objective. Full z-stacks were acquired for analysis. 


\section{RNAscope analysis}

RGL-NSCs and astrocytes were identified based on GFAP+ apical or stellate processes, respectively, extending from a Hoechst+ nucleus in $1 \mu \mathrm{m}$ z-stack images from $n=4$ mice. Vegfa puncta were found almost exclusively in the nucleus in both cell types and were counted manually throughout the depth of the nucleus. Kdr puncta were also counted within RGL-NSC Hoechst+ nuclei.

\section{In vitro Treatment}

SU5416 and SU1498 (Sigma) were dissolved in DMSO (10 mM) and stored at $-20^{\circ} \mathrm{C}$ until use. SU5416 was used at $25 \mu \mathrm{M}$ unless otherwise noted and SU1498 was used at 2.5 $\mu \mathrm{M}$. VEGF nAb in sterile PBS (B20-4.1.1) was provided by Genentech Inc. (Pan et al., 2007) and used at $10 \mu \mathrm{g} / \mathrm{ml}$ unless otherwise noted. Mouse recombinant VEGF164 (R\&D Systems) was dissolved in sterile PBS and stored at $-20^{\circ} \mathrm{C}$. It was used at $10 \mathrm{ng} / \mathrm{ml}$ unless otherwise noted.

\section{Immunoblotting}

Phosphorylation of PI3K/Akt and MAPK/Erk pathways were used as indicators of VEGFR2 signaling because they are both major downstream targets of VEGFR2 activity and because we found commercial pVEGFR2 antibodies to be unreliable, even in cells with known high levels of pVEGFR2 activity such as bEnd.3 cells (data not shown). NSCs were treated at $\sim 70 \%$ confluency. Confluent HUVECs were serum-starved for 6h before treatment. NSC are HUVEC monolayers were lysed with $65 \mu$ RIPA buffer (Pierce) with 1x Halt Protease and Phosphatase Inhibitor (Thermo Scientific) on ice for $10 \mathrm{~min}$. Scraped lysates were freeze-thawed 3 times and then centrifuged at $14000 \mathrm{rpm}$ for $10 \mathrm{~min}$ at $4^{\circ} \mathrm{C}$. Total protein concentration of the supernatant was quantified using a BCA kit (Pierce). Lysates were run on 4-12\% bis-tris gels in 1x NuPage MOPS buffer (Invitrogen) at 120 $\mathrm{V}$ for $2 \mathrm{~h}$. Gels were transferred to nitrocellulose membrane overnight in $20 \%$ methanol in NuPage Transfer Buffer (Invitrogen) at $4^{\circ} \mathrm{C}$. Membranes were blocked in $5 \%$ milk in $0.1 \mathrm{M}$ tris buffered saline with $0.5 \%$ Tween20 (TBS-t) then cut into weight appropriate strips (using standard ladder markers and predicted molecular weights) for simultaneous probing of pAkt/Akt and pErk/Erk. Strips were incubated in primary antibody overnight at $4^{\circ} \mathrm{C}$ in 5\% milk in TBS-t. Secondary was applied for $1 \mathrm{~h}$ at room temperature in $5 \%$ milk in TBS-t. Proteins were visualized and quantified on a LICOR Odyssey infrared imaging system. Quantification was taken as the ratio of phosphorylated protein to its unphosphorylated form.

\section{Lentiviral vector production}

mCherry-Cre (Addgene no. 27546) and mCherry-only vectors are described in Kirby et al., 2015. Plasmids were packaged in vesicular stomatitis virus-glycoprotein G (VSV-G) lentivirus by the Cincinnati Children's Hospital Viral Vector Core. VEGF and scramble shRNA lentiviral vectors are described in Mosher et al., 2012 and were packaged in VSV-G lentivirus by either Vigene Biosciences or the Stanford Gene Vector and Virus Core.

\section{Neighbor rescue experiment}

Adult DG NSCs were grown in standard media on uncoated, tissue culture plates, allowing them to form spheres. On day 1,5000 NSCs derived from DG of 6-week-old VEGFloxlox mice or VEGFwtwt were infected with mCherry-Cre or mCherry-only lentiviral vectors titered to result in $<5 \%$ total mCherry+ cells. 4 days after infection, short term neighbor rescue experiment NSCs were incubated with $20 \mu \mathrm{M}$ 5-bromo-2'-deoxyuridine (BrdU) (Sigma) for 2 hours then fixed with 4\% paraformaldehyde prior to immunohistochemical processing. Long term neighbor rescue NSC spheres were dissociated every $4 \mathrm{~d}$ and freshly plated single cells were imaged live to quantify the percent of mCherry+ cells among those identified by brightfield contrast. Images were quantified by a blinded observer.

\section{VEGF ELISA of in vitro VEGFlox/lox conditioned media}

Adult DG VEGFloxlox NSCs were infected with mCherry-Cre or mCherry-only lentiviral vectors titered to result in $<5 \%$ mCherry+ cells (low) or $>50 \%$ mCherry+ cells (high). After $4 \mathrm{~d}$, cells and conditioned media were collected and centrifuged $1000 \mathrm{~g}$ for $5 \mathrm{~min}$. Conditioned media supernatant was extracted and processed with the Mouse VEGF DuoSet ELISA (R\&D Systems, DY493-05) according to manufacturer instructions.

\section{shRNA Lentiviral Infection in vitro}

Adult Wt C57/BI6JJ mouse DG derived NSCs were plated on coated 96-well plates at a density of 10,000 cells/well then infected with lentiviral vectors containing a VEGF shRNA or a scramble shRNA (described in Mosher et al., 2012) at a MOI of 50. Media was changed after 24h. After $3 \mathrm{~d}$, NSPCs were transferred to sphere 
bioRxiv preprint doi: https://doi.org/10.1101/2021.08.10.455866; this version posted August 10,2021. The copyright holder for this preprint (which was not certified by peer review) is the author/funder, who has granted bioRxiv a license to display the preprint in perpetuity. It is made available under aCC-BY-NC 4.0 International license.

conditions on uncoated plates at 5000 cells/well and fed FGF2/EGF every 2d. Sphere number and size was quantified using a CellAvista automated microscope system (Roche). Conditioned media was collected from supernatant at first passage and assayed with the Mouse VEGF DuoSet ELISA (R\&D Systems, DY493-05) according to manufacturer instructions.

\section{Stereotaxic Surgery}

Mice were anesthetized by inhalation of isoflurane (Akorn, $5 \%$ induction, 1-2\% maintenance) in oxygen and mounted in the stereotaxic apparatus (Stoelting). Ocular lubricant (Puralube) was placed over the eyes to prevent evaporative dry eye. Following sterilization with alcohol (Fisher) and betadine swabs (Fisher), the skull was exposed and the lambda and bregma sutures were aligned in the same horizontal plane. A small bur hole was drilled in the skull and an automated injector (Stoelting) with a Hamilton syringe (Hamilton) was lowered to the injection depth at a rate of $-1.0 \mathrm{~mm} / \mathrm{min}$. Mice were injected with $0.5 \mu \mathrm{L}$ of scramble control shRNA virus into one hemisphere and $0.5 \mu \mathrm{L}$ of Vegfa shRNA virus into the contralateral hemisphere at a rate of $0.1 \mu \mathrm{L} / \mathrm{min}$. The injection coordinates from bregma were: anterior/posterior $-1.9 \mathrm{~mm}$, medial/lateral $\pm 1.6 \mathrm{~mm},-1.9 \mathrm{~mm}$ dorsal/ventral from dura. Post-surgery, the incision was sealed with tissue adhesive (3M) and the mouse was given saline (Hospira) and carprofen (Zoetis) injection ip. After 21 or $60 \mathrm{~d}$, mice were sacrificed for immunohistochemical processing.

\section{EdU and BrdU labeling}

After 21 or 60 days, mice were injected with 5-Ethynyl-2'-deoxyuridine (EdU) (Click Chemistry Tools) dissolved in physiological saline (Hospira) $(150 \mathrm{mg} / \mathrm{kg}, \mathrm{IP})$ and sacrificed 2 hours later for tissue processing. 4 weeks after viral infusion (i.e. 4 weeks before perfusion), the $60 \mathrm{~d}$ cohort received 5 daily i.p. injections of 5-bromo-2'deoxyuridine (BrdU, 1/day over 5 days) (Sigma).

\section{Immunohistochemical processing}

\section{Cultured NSCs}

Adherent NSCs were fixed with 4\% paraformaldehyde then rinsed with PBS 3x before incubating in a blocking solution containing $1 \%$ normal donkey serum and $0.3 \%$ Triton X-100 (Acros) in PBS. Cells were then incubated in primary antibody diluted in blocking solution overnight at $4{ }^{\circ} \mathrm{C}$. The following day, after 3 rinses in PBS, cells were incubated in secondary antibodies diluted in blocking solution for 2 hours. If a biotinylated secondary was used, a fluorophore-conjugated tertiary was applied for $1 \mathrm{~h}$ before rinsing an nuclei counterstaining with Hoechst (10 min, 1:2000 in PBC) (Invitrogen). If proceeding for BrdU labeling, the cells were rinsed and fixed with 4\% paraformaldehyde in $0.1 \mathrm{M} \mathrm{PB}$ for 10 min, rinsed with PBS $3 \mathrm{x}$ and incubated with $2 \mathrm{~N} \mathrm{HCl}$ for 30 min at $37^{\circ} \mathrm{C}$. After 3 PBS rinses and 30 min incubation in blocking, cells were incubated in BrdU primary antibody diluted in blocking solution overnight at $4{ }^{\circ} \mathrm{C}$. The next day, cells were rinsed $3 x$ with PBS and exposed to a secondary antibody diluted in blocking solution for $2 \mathrm{~h}$ before Hoechst counterlabeling. Cells in 96 -well plates were imaged immersed in PBS using 10x magnification while those on chamber slides were coverslipped with Prolong Gold Antifade Mountant (Fisher, P36934) and imaged using 40x oil magnification, both with a Zeiss apotome digital imaging system (Zeiss).

\section{Brain Sections}

Brains for immunolabeling were harvested following perfusion with ice-cold PBS followed by fixation in $4 \%$ paraformaldehyde overnight at $4{ }^{\circ} \mathrm{C}$. After equilibration in $30 \%$ sucrose in PBS, $40 \mu \mathrm{m}$ coronal brain sections were obtained in 1 in 12 series on a freezing microtome (Leica) and stored in cryoprotectant at $-20{ }^{\circ} \mathrm{C}$. Sections were rinsed $3 x$ in PBS and incubated in a blocking solution containing $1 \%$ normal donkey serum and $0.3 \%$ Triton X-100 (Acros) in PBS before incubation in primary antibodies. The next day, cells were rinsed $3 x$ with PBS and exposed to a secondary antibody diluted in blocking solution for $2 \mathrm{~h}$. EdU labeling was performed according to manufacturer recommendations for using fixed frozen tissue samples to enable concurrent immunohistochemical staining (Click Chemistry Tools) before the immunolabeling protocol. BrdU immunolabeling was performed after other immunolabeling was complete. Labeled sections were fixed in $4 \%$ paraformaldehyde for $10 \mathrm{~min}$ at room temperature, rinsed and then incubated with $2 \mathrm{~N} \mathrm{HCl}$ for 30 min at $37^{\circ} \mathrm{C}$. Sections were then rinsed, blocked and incubated in anti-BrdU primary and appropriate secondaries as above. The DG of the hippocampus was imaged in $15 \mu \mathrm{m}$ Z-stacks at 20x magnification using a Zeiss apotome digital imaging system (Zeiss).

\section{NSPC identification and DG cell counts}


NSC-RGLs were identified by GFAP+/Sox2+ colocalization and radial processes extending from the SGZ towards the inner molecular layer, while GFAP-/Sox2+ cells in the SGZ layer were identified as IPCs. EdU+ cells were identified as an EdU+ nucleus in the subgranular zone. BrdU+ nuclei were counted in the subgranular zone and granular cell layer. Cell density in all cases was determined as the number of cells per area in the DG. The SGZ was defined as the zone spanning 2 cell body widths between the dense granular cell layer and the hilus. Cell counts were performed manually in Zen by a blind observer.

\section{CRISPRi VEGFR2 knockdown for immunolabeling}

bEnd.3 cells or NSCs were plated on uncoated (bEnd.3) or on PDL/laminin-coated (NSCs) glass 8-chamber slides (Fisher) and allowed to adhere for $24 \mathrm{~h}$. After $24 \mathrm{~h}$, cells were transfected with CRISPRi expressing plasmids using Lipofectamine 2000 (Fisher), according to manufacturer instructions. Media was changed to remove lipofectamine/DNA $5 \mathrm{~h}$ later and replaced with standard growth media for each respective cell type. Two days later, cells were fixed with $4 \%$ paraformaldehyde.

\section{Plasmid construction and sgRNA design}

The CRISPR interference (CRISPRi) lentivirus construct was modified from pLV hU6-sgRNA hUbC-dCas9KRAB-T2a-GFP (Addgene \#71237, a gift from Charles Gersbach, Thakore et al., 2015), which expresses all necessary CRISPRi machinery (both the dCas9-KRAB and sgRNA) from the same plasmid. The UbC promoter was replaced with a Nestin regulatory element comprised of the Nestin promoter and second intronic enhancer from (Keyoung et al., 2001) to create pLV hU6-sgRNA Nestin-dCas9-KRAB-T2a-GFP. Additionally, two Esp3I recognition sites were placed after the U6 promoter for restriction cloning of sgRNA insert sequences. The insert sequences were synthesized as single strand oligonucleotides (Integrated DNA Technologies) in the form of 5 ' GGACG $(N)_{20}$ 3' and 5' AAAC $\left(N^{\prime}\right)_{20}$ C 3' where $(N)_{20}$ refers to the sequence of the sgRNA and ( $\left.{ }^{\prime}\right)_{20}$ is the reverse complement. These oligonucleotides were annealed together and ligated with Esp3I-digested pLV hU6-sgRNA Nestin-dCas9-KRAB-T2a-GFP to obtain the final constructs for lentivirus packaging. The sgRNAs targeting the region -50 to +300 bp relative to the transcriptional start sequence of Kdr were designed using the CRISPRi function of the Broad Institute GPP portal (https://portals.broadinstitute.org/gpp/public/analysis-tools/sgrnadesign). The top 5 ranked sgRNAs returned by the GPP tool were chosen and a non-targeting sgRNA (Chiou et al., 2015) was used as control.

\section{VEGFR2 cell surface and intracellular immunolabeling and quantification}

To label all VEGFR2, blocking solution was $1 \%$ normal donkey serum, $0.3 \%$ tritonX 100 in PBS. To label only cell surface VEGFR2, the triton 100X was omitted but all other procedures were identical. 3 random view fields were captured for VEGFR2 intensity quantification using thresholded area and intensity within cell bodies using Image J.

\section{TAPI1 treatment}

NSCs derived from 6-week-old Wt C57BI6J DG were plated at 20k cell/well on PDL/laminin-coated glass 8chamber slides (Fisher 08-774-26) and allowed to adhere for 24h. TAPI1 (Fisher 61-621) was added to media at $0-80 \mu \mathrm{g} / \mathrm{ml}$. NSCs were fixed $24 \mathrm{~h}$ later.

\section{CRISPRi VEGFR2 knockdown for qPCR}

bEnd.3 cells were plated on 12-well tissue culture plates (VWR \#82050-930) and allowed to grow to 80\% confluency before transfection with CRISPRi plasmids using Lipofectamine 2000 (Fisher L3000001), according to manufacturer instructions. 18h later, cells were harvested by scraping in $0.25 \%$ Tryspin-EDTA with phenol red (Fisher 25-200-056), then centrifuged $1000 \mathrm{~g}$ for $5 \mathrm{~min}$. RNA was isolated from cell pellets using the Aurum Total RNA Mini kit (Bio-Rad, 732-6820). RNA was quantified using a BioTek Epoch Microplate Spectrophotometer and 250 ng RNA was converted to cDNA using the iScript cDNA synthesis kit (Bio-Rad 1708891) and a ThermoFisher Applied Biosystems 2720 Thermal Cycler. cDNA was reacted with gene-specific primers and SsoAdvanced Universal SYBR Green Supermix in a Bio Rad CFX96 Touch Real-Time PCR Detection System. Melt curves were used to confirm purity of the amplified product and Ct value was normalized to houskeeping gene Hprt. $\Delta \Delta \mathrm{Ct}$ values were used to obtain fold change over non-target control average.

Hprt: PrimerBank ID: 96975137c1

Forward primer: agtcccagcgtcgtgattag

Reverse primer: tttccaaatcctcggcataatga

Kdr: From Kirby et al., 2015 
Forward primer: atctttggtggaagccacag

Reverse primer: ccatgatggtgagttcatcg

\section{Diagram creation}

All diagrams, including the graphical abstract, in this manuscript were created with BioRender.com.

\section{Quantification and Statistical Analysis}

Statistics were performed as described in each figure legend. Western blots for Akt and Erk phosphorylation after SU5416, VEGF nAb or recombinant VEGF treatment were compared to control using One-way ANOVA with Dunnett's multiple comparison test. RGL-NSC and astrocyte expression of Vegfa were compared using the Kolmogorov-Smirnov test for individual cells or t-test when comparing mean expression per cell. Two-way ANOVAs were used to compare the effect of mCherry-Cre and mCherry in the Neighbor Rescue Experiment with Tukey's or Sidak's multiple comparison post hoc tests. VEGF immunoreactivity was compared by paired ttest. Three-way ANOVAs were used to compare virus $x$ day $x$ GFP expression interaction in viral infected tissue sections. After confirmation of significant 3-way interaction, two-way ANOVAs were used within GFP+ or GFPcell populations to confirm a significant virus $x$ day interaction followed by Sidak's multiple comparisons post hoc tests. The effect of viral shRNA expression on differentiation was assessed using two-way ANOVA. The effect of TAPI-1 on VEGFR2 immunoreactivity was compared to control by One-way ANOVA with Kruskal-Wallis test. All analyses were performed using Prism (v9.0; GraphPad Software) and $p<0.05$ was considered significant.

\section{Key Resources Table}

\begin{tabular}{|c|c|c|}
\hline REAGENT or RESOURCE & SOURCE & IDENTIFIER \\
\hline \multicolumn{3}{|l|}{ Antibodies } \\
\hline Cell Signaling Technology, 2920s, Akt (pan) & $\begin{array}{l}\text { Cell Signaling } \\
\text { Technology }\end{array}$ & $\begin{array}{l}\text { Cat\#2920 } \\
\text { RRID: AB_1147620 }\end{array}$ \\
\hline Cell Signaling Technology phospho-Akt (ser473) & $\begin{array}{l}\text { Cell Signaling } \\
\text { Technology }\end{array}$ & $\begin{array}{l}\text { Cat\#4060 } \\
\text { RRID: AB_2315049 }\end{array}$ \\
\hline $\begin{array}{l}\text { Cell Signaling Technology, 9107s, p44/42 MAPK } \\
(\text { Erk1/2) }\end{array}$ & $\begin{array}{l}\text { Cell Signaling } \\
\text { Technology }\end{array}$ & $\begin{array}{l}\text { Cat\#9107 } \\
\text { RRID: AB_10695739 }\end{array}$ \\
\hline $\begin{array}{l}\text { Cell Signaling Technology phospho-p44/42 MAPK } \\
\text { (Erk1/2) }\end{array}$ & $\begin{array}{l}\text { Cell Signaling } \\
\text { Technology }\end{array}$ & $\begin{array}{l}\text { Cat\#9106 } \\
\text { RRID: AB_331768 }\end{array}$ \\
\hline Hoechst 33342 & Fisher & $\begin{array}{l}\text { Cat\#H3570 } \\
\text { RRID: }\end{array}$ \\
\hline Anti-Glial Fibrillary Acidic Protein, Clone: GA5 & Sigma & $\begin{array}{l}\text { Cat\#MAB360 } \\
\text { RRID: AB_11212597 }\end{array}$ \\
\hline Anti-mCherry antibody & Abcam & $\begin{array}{l}\text { Cat\#ab167453 } \\
\text { RRID: AB_2571870 }\end{array}$ \\
\hline GFP Tag Polyclonal Antibody & BD Biosciences & $\begin{array}{l}\text { Cat\#A11122 } \\
\text { RRID: AB } 221569\end{array}$ \\
\hline BrdU Antibody & Bio-Rad & $\begin{array}{l}\text { Cat\# MCA6114 } \\
\text { RRID: }\end{array}$ \\
\hline Goat anti-VEGF164 & R\&D systems & $\begin{array}{l}\text { Cat\#AF-493 } \\
\text { RRID: AB_354506 }\end{array}$ \\
\hline SOX2 Rat anti-Human, Mouse, Clone: Btjce & eBioscience & $\begin{array}{l}\text { Cat\# 50-112-9095 } \\
\text { RRID: }\end{array}$ \\
\hline Anti-NeuN, clone A60 & Millipore & $\begin{array}{l}\text { Cat\#MAB377 } \\
\text { RRID: }\end{array}$ \\
\hline Rat anti-VEGFR2 & R\&D systems & $\begin{array}{l}\text { Cat\#MAB4432 } \\
\text { RRID: AB } 2298772\end{array}$ \\
\hline $\begin{array}{l}\text { Donkey anti-Goat lgG }(\mathrm{H}+\mathrm{L}) \text { Cross-Adsorbed Secondary } \\
\text { Antibody, Alexa Fluor } 488\end{array}$ & Fisher & $\begin{array}{l}\text { Cat\#A-11055 } \\
\text { RRID: AB_2534102 }\end{array}$ \\
\hline $\begin{array}{l}\text { Donkey anti-Rabbit IgG }(\mathrm{H}+\mathrm{L}) \text { Highly Cross-Adsorbed } \\
\text { Secondary Antibody, Alexa Fluor } 555\end{array}$ & Fisher & $\begin{array}{l}\text { Cat\#A-31572 } \\
\text { RRID: AB } 162543\end{array}$ \\
\hline $\begin{array}{l}\text { Donkey anti-Goat lgG }(\mathrm{H}+\mathrm{L}) \text { Cross-Adsorbed Secondary } \\
\text { Antibody, Alexa Fluor } 555\end{array}$ & Fisher & $\begin{array}{l}\text { Cat\#A-21432 } \\
\text { RRID: AB_2535853 }\end{array}$ \\
\hline $\begin{array}{l}\text { Donkey anti-Mouse IgG }(\mathrm{H}+\mathrm{L}) \text { Highly Cross-Adsorbed } \\
\text { Secondary Antibody, Alexa Fluor } 647\end{array}$ & Fisher & $\begin{array}{l}\text { Cat\#A-21447 } \\
\text { RRID: AB_141844 }\end{array}$ \\
\hline
\end{tabular}


bioRxiv preprint doi: https://doi.org/10.1101/2021.08.10.455866; this version posted Auqust 10,2021. The copyright holder for this preprint (which was not certified by peer review) is the author/funder, who has granted bioRxiv a license to display the preprint in perpetuity. It is made available under aCC-BY-NC 4.0 International license.

\begin{tabular}{|c|c|c|}
\hline $\begin{array}{l}\text { Donkey anti-Rat IgG }(\mathrm{H}+\mathrm{L}) \text { Highly Cross-Adsorbed } \\
\text { Secondary Antibody, Alexa Fluor } 594\end{array}$ & Fisher & $\begin{array}{l}\text { Cat\#A-21209 } \\
\text { RRID: AB_2535795 }\end{array}$ \\
\hline $\begin{array}{l}\text { IgG }(\mathrm{H}+\mathrm{L}) \text { Highly Cross-Adsorbed Donkey anti-Rabbit, } \\
\text { Alexa Fluor }{ }^{\mathrm{TM}} 350\end{array}$ & Fisher & $\begin{array}{l}\text { Cat\#A-10039 } \\
\text { RRID: AB } 2534015\end{array}$ \\
\hline \multicolumn{3}{|l|}{ Chemicals, peptides, and recombinant proteins } \\
\hline SU5416 & Sigma & Cat\# S8442 \\
\hline SU1498 & Sigma & Cat\# SML1193 \\
\hline VEGF nAb & Genentech Inc & Pan et al., 2007 \\
\hline Mouse recombinant VEGF164 & R\&D Systems & Cat\# AF493SP \\
\hline 5-bromo-2'-deoxyuridine & Sigma & Cat\# B5002 \\
\hline 5-Ethynyl-2'-deoxyuridine & $\begin{array}{l}\text { Click Chemistry Tool } \\
\text { Kit }\end{array}$ & Cat\#1324 \\
\hline TAPI1 & Fisher & Cat\#61-621 \\
\hline \multicolumn{3}{|l|}{ Critical commercial assays } \\
\hline RNAscope Multiplex Fluorescent Assay v2 & ACD & Cat\# 323136 \\
\hline Mouse VEGF DuoSet ELISA & R\&D Systems & Cat\# DY493-05 \\
\hline Click-\&-Go EdU 647 Cell Proliferation Assay Kit & $\begin{array}{l}\text { Click Chemistry Tool } \\
\text { Kit }\end{array}$ & Cat\# 1329 \\
\hline \multicolumn{3}{|l|}{ Deposited data } \\
\hline \multicolumn{3}{|l|}{ CRISPRi NT } \\
\hline \multicolumn{3}{|l|}{ CRISPRi $\alpha$ VEGFR2 } \\
\hline \multicolumn{3}{|l|}{ Experimental models: Cell lines } \\
\hline Cultured Neural Stem Cells & Babu et al., 2011 & $\begin{array}{l}\text { doi: } \\
10.3389 / \text { fnins.2011.0 } \\
0089\end{array}$ \\
\hline bEnd.3s & ATCC & $\begin{array}{l}\text { Cat\#2299 } \\
\text { RRID: }\end{array}$ \\
\hline HUVECs & Invitrogen & $\begin{array}{l}\text { Cat\# C0035C } \\
\text { RRID: }\end{array}$ \\
\hline \multicolumn{3}{|l|}{ Experimental models: Organisms/strains } \\
\hline \multicolumn{3}{|l|}{ Wild type Mice } \\
\hline \multicolumn{3}{|l|}{ VEGFlox/lox Mice } \\
\hline \multicolumn{3}{|l|}{ Software and algorithms } \\
\hline ImageJ & schneider & $\begin{array}{l}\text { https://imagej.nih.go } \\
\text { v/ij/ }\end{array}$ \\
\hline Zen & Zeiss & $\begin{array}{l}\text { https://www.zeiss.co } \\
\text { m/microscopy/us/pro } \\
\text { ducts/microscope- } \\
\text { software/zen.html }\end{array}$ \\
\hline Prism & GfaphPad & $\begin{array}{l}\text { https://www.graphpa } \\
\text { d.com/scientific- } \\
\text { software/prism/ }\end{array}$ \\
\hline \multicolumn{3}{|l|}{ Other } \\
\hline Batiuk et al., 2020 & & $\begin{array}{l}\text { doi: } \\
\text { 10.1038/s41467- } \\
019-14198-8\end{array}$ \\
\hline Hochgerner et al., 2018 & & $\begin{array}{l}\text { doi: } \\
\text { 10.1038/s41593- } \\
017-0056-2\end{array}$ \\
\hline Walker et al., 2020 & & $\begin{array}{l}\text { doi: } \\
\text { 10.1186/s12967- } \\
020-02247-6\end{array}$ \\
\hline Denninger et al., 2020 & & $\begin{array}{l}\text { doi: } \\
\text { 10.1016/j.brainres.2 } \\
020.146717\end{array}$ \\
\hline
\end{tabular}


bioRxiv preprint doi: https://doi.org/10.1101/2021.08.10.455866; this version posted August 10, 2021. The copyright holder for this preprint (which was not certified by peer review) is the author/funder, who has granted bioRxiv a license to display the preprint in perpetuity. It is made available under aCC-BY-NC 4.0 International license.

Shin et al., 2015

doi:

10.1016/j.stem.2015.

07.013 


\section{Supplement Figures}

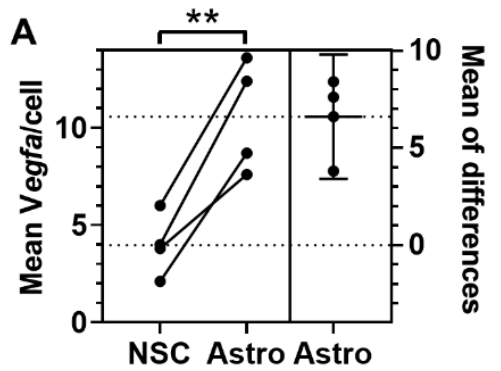

NSC

D

Berg et al., 2019

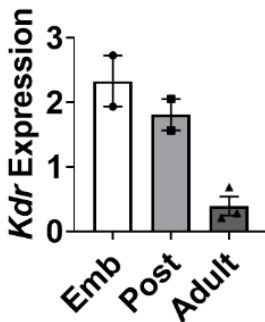

Hopx+ NSCs
B

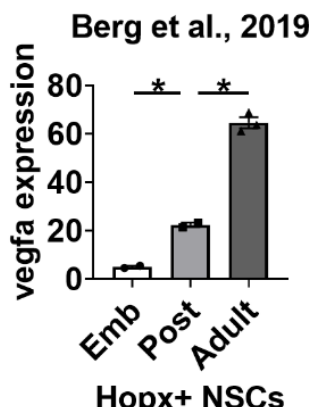

E

Adusumilli et al., 2021

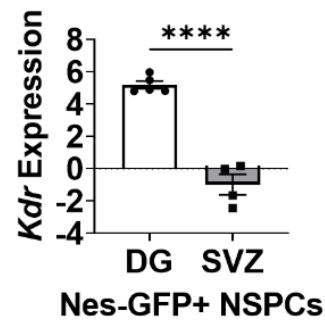

Nes-GFP+ NSPCs
C Adusumilli et al., 2021

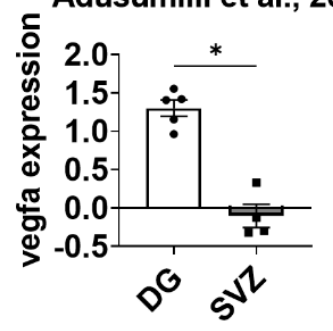

Nes-GFP+ NSPCs

$\mathbf{F}$

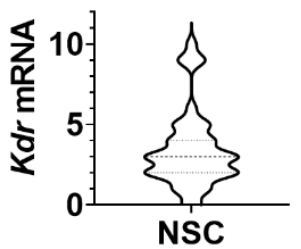

NSC
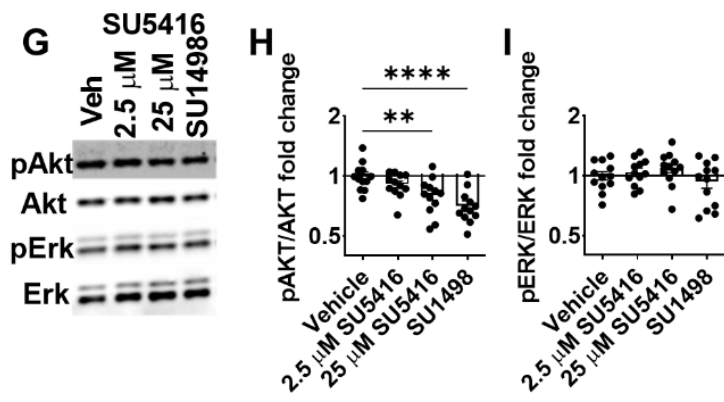

$\mathrm{J}_{\mu \mathrm{g} / \mathrm{ml} \text { VEGF nAb } \mathrm{g}} \mathrm{K}$

011050
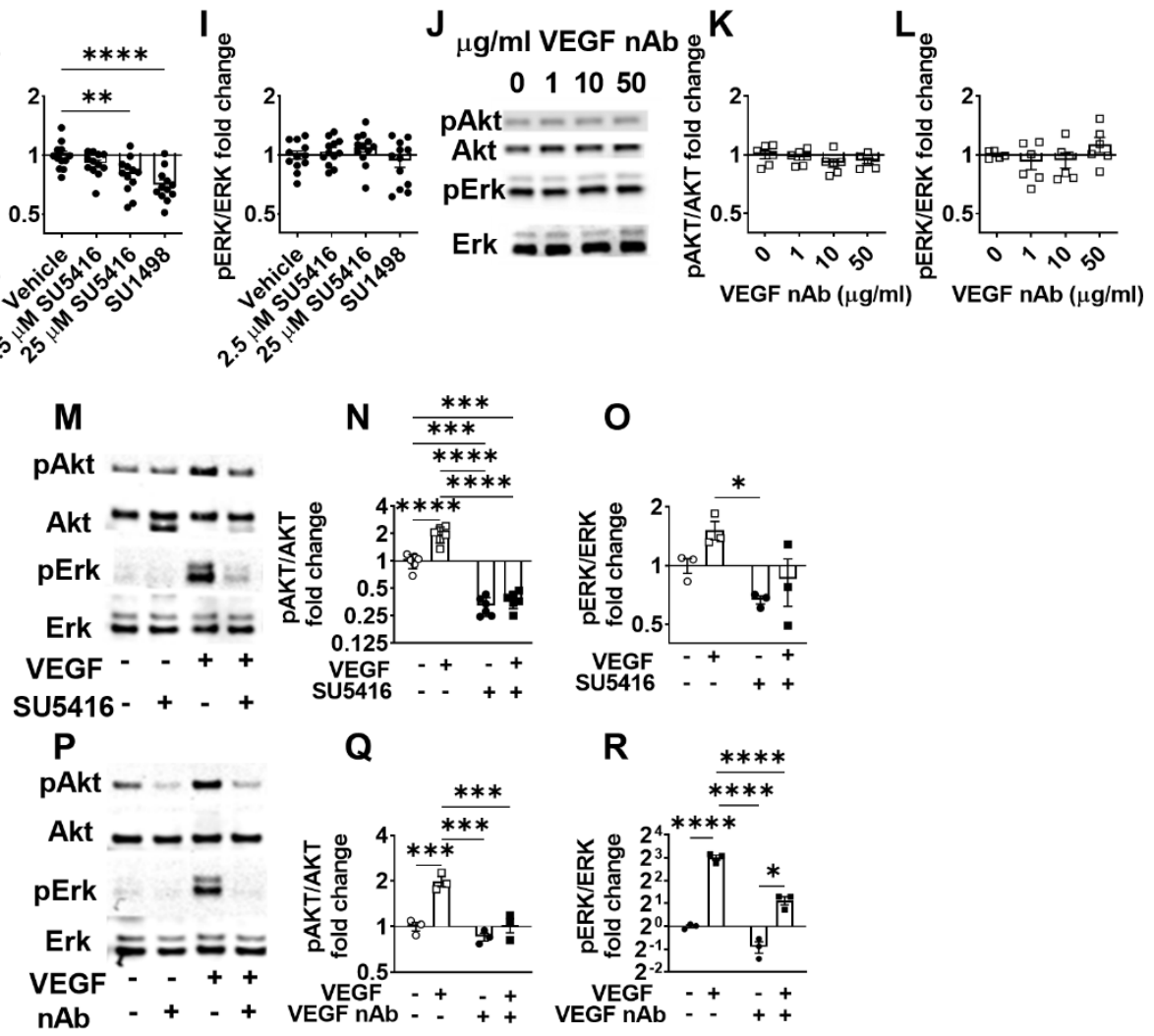

Figure S1: NSC-VEGF expression is specific to the adult DG, and signals cell-autonomously through VEGFR2, related to Figure 1.

A) Mean Vegfa RNA per cell in RGL-NSCs and Astrocytes. $\mathrm{N}=4$ mice; mean \pm SEM. Paired t-test

B) Vegfa expression across development in Hopx+ NSCs isolated from mouse DG and quantified via single-cell RNA sequencing originally published in Berg et al., 2019. $\mathrm{N}=2-3$ replicates; mean \pm SEM. One-way ANOVA with Dunnett's multiple comparison test.

C) Vegfa expression of Nes-GFP+ NSPCs in the DG or SVZ quantified via bulk RNA sequencing originally published in Adusumilli et al., 2021. N = 4-5 replicates; mean \pm SEM. One-way ANOVA with Dunnett's multiple comparison test. 
D) $K d r$ expression across development in Hopx+ NSCs isolated from mouse DG and quantified via single-cell RNA sequencing originally published in Berg et al., 2019. $\mathrm{N}=2-3$ replicates; mean \pm SEM. One-way ANOVA with Dunnett's multiple comparison test.

E) Kdr expression of Nes-GFP+ NSCs in the DG or SVZ quantified via bulk RNA sequencing originally published in Adusumilli et al., 2021. $N=4-5$ replicates; mean \pm SEM. One-way ANOVA with Dunnett's multiple comparison test.

F) Kdr RNA expression within RGL-NSCs of the adult DG by puncta count/cell in RNAscope in situ hybridization. $\mathrm{N}=40$ cells; mean \pm SEM.

G) Representative western blot of Erk and Akt phosphorylation in cultured DG NSCs after treatment with SU5416 or SU1498.

H) Fold change in Akt or I) Erk phosphorylation after SU5416 and SU1498 treatment in cultured NSCs. N = 34/grp/exp, 3 exps; mean \pm SEM. One-way ANOVA with Dunnett's multiple comparison test.

J) Representative western blot of Erk and Akt phosphorylation in cultured DG NSCs after treatment with VEGF nAb.

K) Fold change in Akt or L) Erk phosphorylation after VEGF nAb treatment in cultured NSCs. N = 2-3/grp/exp, 3 exps; mean \pm SEM. One-way ANOVA with Dunnett's multiple comparison test.

M) Representative western blot showing of Erk and Akt phosphorylation in cultured HUVECs after treatment with recombinant mouse VEGF and SU5416.

N) Fold change in Akt or O) Erk phosphorylation after VEGF and SU5416 treatment in cultured HUVECs. N = 23/grp/exp, 1-2 exps; mean \pm SEM. Two-way ANOVA with Tukey's multiple comparisons test.

P) Representative western blot of Erk and Akt phosphorylation in cultured HUVECs after treatment with recombinant VEGF and VEGF nAb

Q) Fold change in Akt or R) Erk phosphorylation after VEGF and VEGF nAb treatment in cultured HUVECs. N = 3/grp/exp, 1 exp; mean \pm SEM. Two-way ANOVA with Tukey's multiple comparisons test.

${ }^{*} p<0.05 ;{ }^{* *} p<0.01 ;{ }^{* * *} p<0.001 ;{ }^{* * * *} p<0.0001$

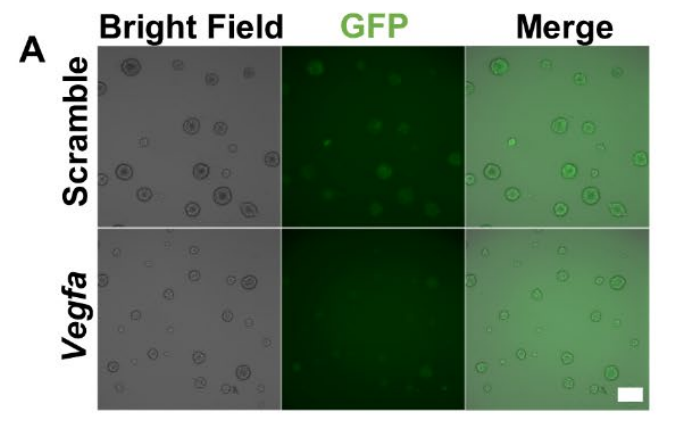

B C
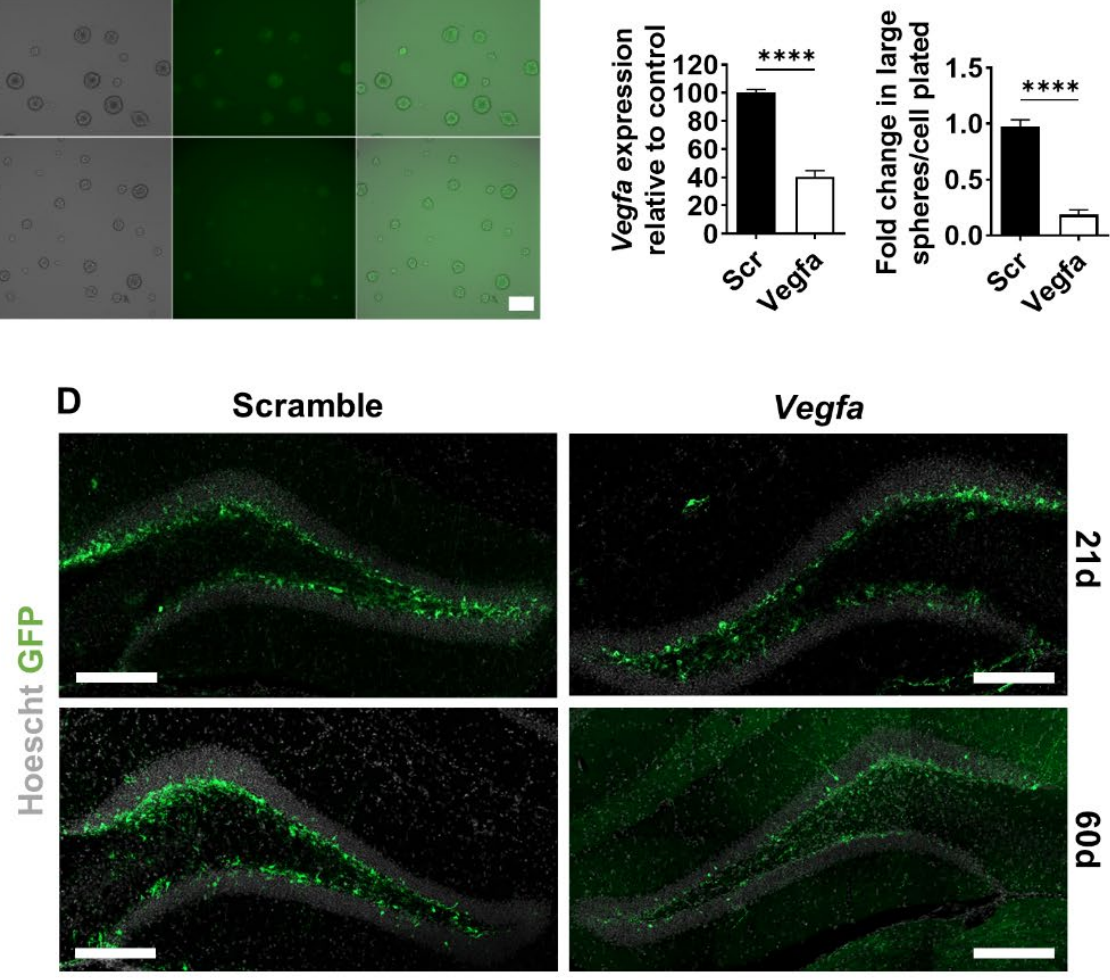

Figure S2: Vegfa shRNA efficacy and lentiviral expression, related to Figure 2. 
A) Representative images of live cultured WT NSC spheres (bright field) after Scramble or Vegfa shRNA lentiviral infection (GFP+).

B) Relative VEGF protein in conditioned media of WT NSCs after Scramble or Vegfa shRNA infection. $\mathrm{N}=$ 3/grp/exp, 2 exps; mean \pm SEM. Unpaired T-test.

C) Fold change in large spheres per cells plated of WT NSCs after Vegfa shRNA infection vs scramble shRNA. $\mathrm{N}=3$ /grp/exp, 2 exps; mean \pm SEM. Unpaired T-test.

D) Representative images of Scramble or Vegfa shRNA infection (GFP+) in the DG (Hoechst) 21 or 60d after viral infusion.

Scale bars represent A) $100 \mu \mathrm{M}$ D) $200 \mu \mathrm{M} .{ }^{* * * *} p<0.0001$;

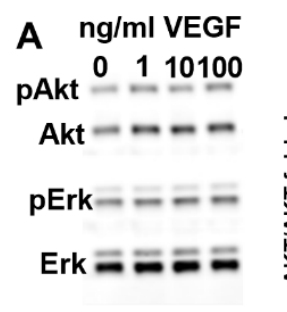

G min after EGF/FGF2 feeding 0153060
pAkt-
Akt ----
pErk $==-$ Erk シニニニ

$\mathbf{L}$

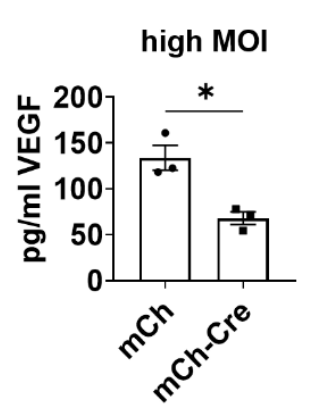

\section{M}
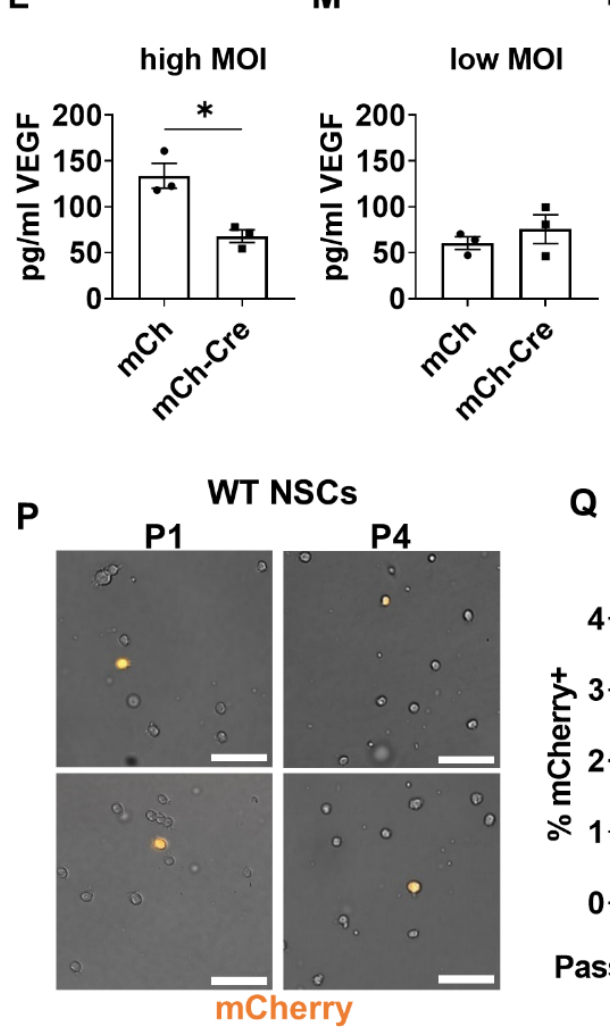

Q
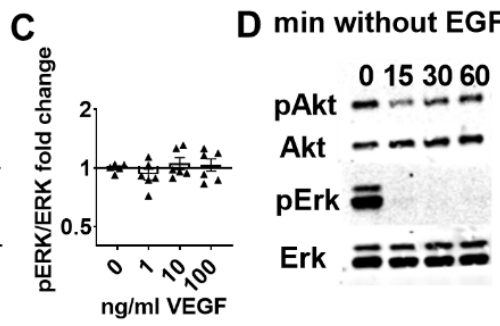

$$
\mathbf{J}
$$

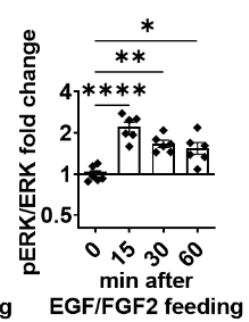

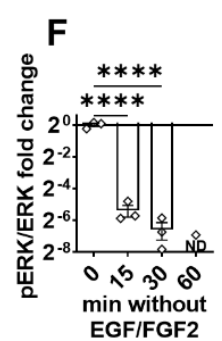

K

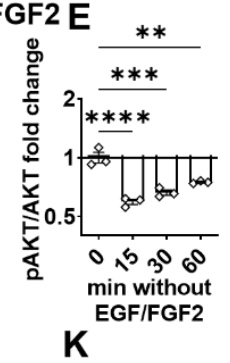

VEGF

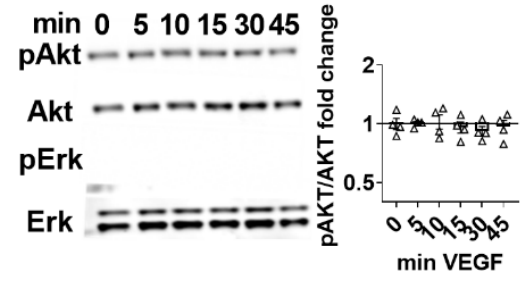

Hoechst mCherry
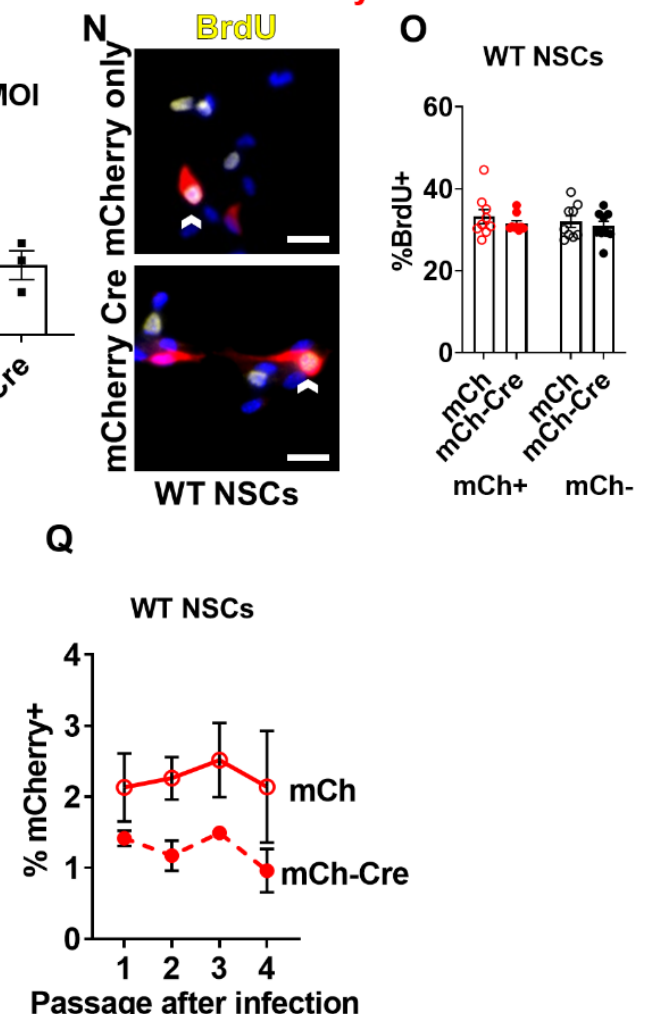

Passage after infection

Figure S3: Lentiviral infecting in WT DG NSCs, related to Figure 3.

A) Representative western blot of Erk and Akt phosphorylation in cultured NSCs after treatment with recombinant VEGF.

B) Fold change in Akt or C) Erk phosphorylation after VEGF treatment in cultured NSCs. $N=3 / g r p / e x p, 2$ exps; mean \pm SEM. One-way ANOVA. 
D) Representative western blot of Erk and Akt phosphorylation in cultured NSCs after EGF/FGF2 washout.

E) Fold change in Akt or F) Erk phosphorylation after EGF/FGF2 washout in cultured NSCs. N = 3/grp/exp, 1 exp; mean \pm SEM. One-way ANOVA.

G) Representative western blot of Erk and Akt phosphorylation in cultured NSCs after supplementing with 20 $\mathrm{ng} / \mathrm{ml}$ EGF/FGF2.

$\mathrm{H})$ Fold change in Akt or I) Erk phosphorylation after $20 \mathrm{ng} / \mathrm{ml}$ EGF/FGF2 supplementing in cultured NSCs. $\mathrm{N}=$ 2-3/grp/exp, 2 exps; mean \pm SEM. One-way ANOVA with Dunnett's multiple comparison test..

J) Representative western blot of Erk and Akt phosphorylation in cultured NSCs treated with recombinant VEGF after 30 min EGF/FGF2 depletion.

K) Fold change in Akt phosphorylation following VEGF treatment in cultured NSCs after 30 min EGF/FGF2 depletion. $\mathrm{N}=2$ /grp/exp, 2 exps; mean \pm SEM. One-way ANOVA with Dunnett's multiple comparison test..

$\mathrm{L})$ VEGF concentration (pg/ml) in NSC conditioned media after treatment with high $\mathrm{MOI}$ or $\mathrm{M}$ ) low MOI mCherry or mCherry-Cre lentivirus. $\mathrm{N}=3$ wells/grp; mean \pm SEM. Unpaired T-test.

N) Representative immunofluorescent images of mitotically active (BrdU+) cultured WT NSCs (Hoechst + ) after lentiviral infection (mCherry+). Chevrons indicate BrdU+, mCherry+ Hoechst+ NSCs.

O) Percent of mCherry+ or mCherry- WT NSCs that were BrdU+ after low MOI mCherry-Cre and mCherry-only lentiviral infection. $\mathrm{N}=3$ /grp/exp, 3 exps; mean \pm SEM. Two-way ANOVA with Tukey's multiple comparisons test.

P) Representative images of cultured WT NSCs (brightfield) after lentiviral infection (mCherry+) after 1 and 4 passages.

F) Percent of mCherry+ WT NSCs after infection with low MOI mCherry-Cre or mCherry-only lentiviral vectors. $\mathrm{N}=3$ /grp/exp, 2 exps; mean \pm SEM. Two-way ANOVA with Sidak's multiple comparisons test.

Scale bars represent C) $10 \mu \mathrm{M}$, E) $50 \mu \mathrm{M}{ }^{*} p<0.05 ;{ }^{* *} p<0.01$ 


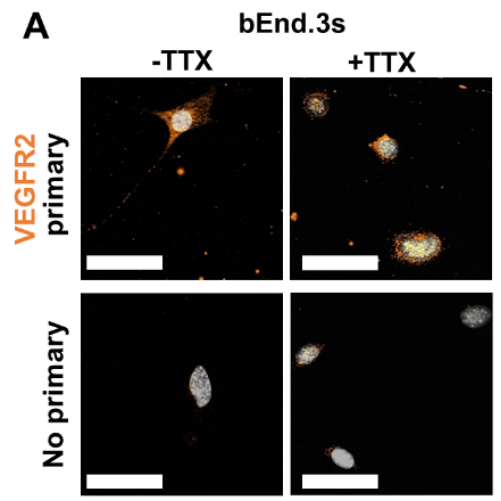

B
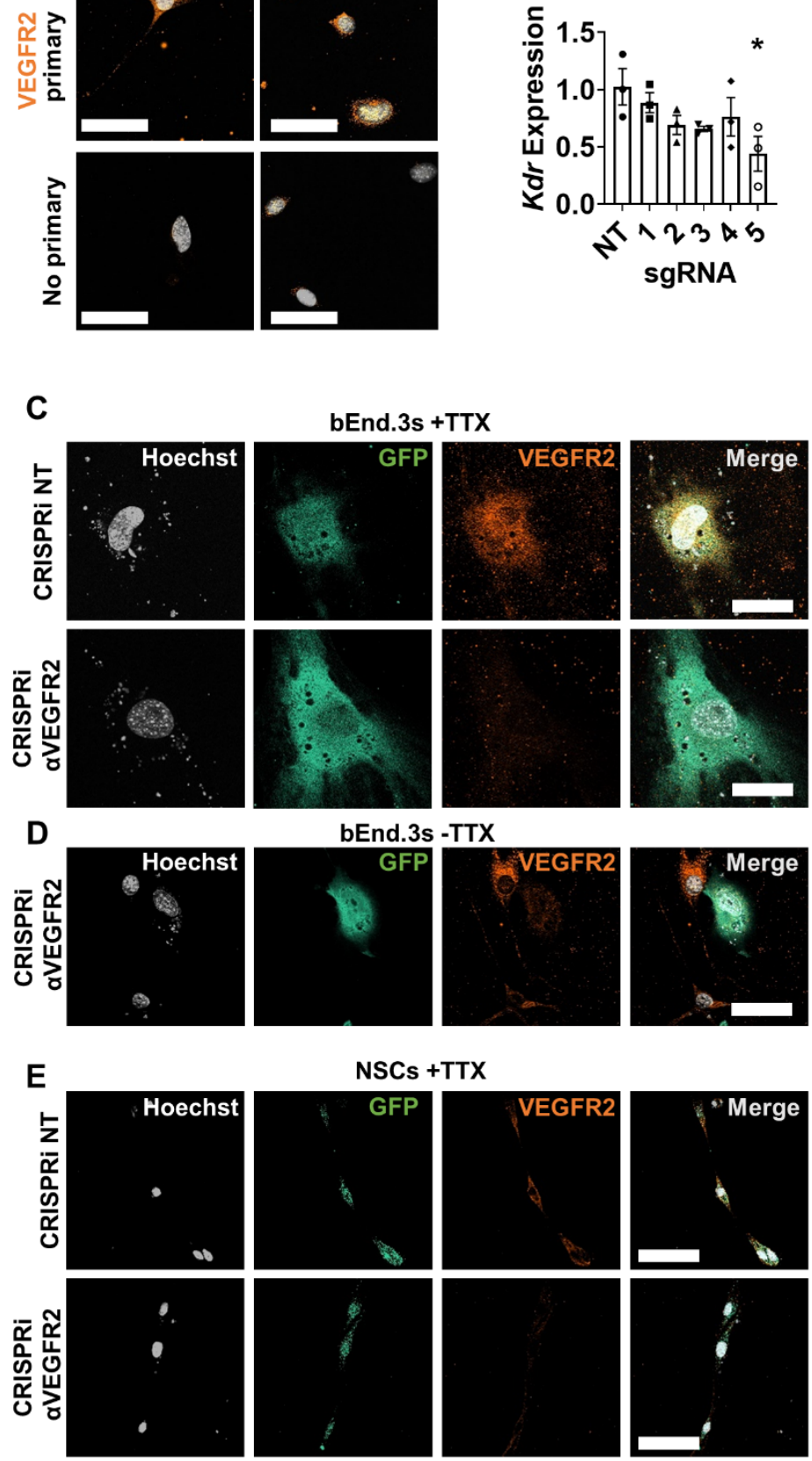

Figure S4: NSCs insensitivity to extracellular VEGF and VEGFR2 antibody confirmation, related to Figure 4.

A) Representative VEGFR2 immunoreactivity in permeabilized and non-permeabilized cultured bEnd.3s in VEGFR2 primary and no primary conditions.

B) $K d r$ expression in bEnd3.s after transfection with plasmids expressing 5 different sgRNAs targeting VEGFR2. $\mathrm{N}=3$ wells/grp/exp, 1 exp; mean \pm SEM. One-way ANOVA with Dunnett's multiple comparison test.

C) Representative images of VEGFR2 immunoreactivity in permeabilized or D) non-permeabilized cultured bEnd.3s following transfection with CRISPRI NT or aVEGFR2.

E) Represetative images of VEGFR2 immunoreactivity in permeabilized cultured NSCs following infection with CRISPRI NT or aVEGFR2.

Scale bars represent $A, C, D, E) 50 \mu M * p<0.05$ 